\title{
MULTIPLIERS OF DIRICHLET SUBSPACES OF THE BLOCH SPACE
}

\author{
CHRISTOS CHATZIFOUNTAS, DANIEL GIRELA, AND JOSÉ ÁNGEL PELÁEZ
}

\begin{abstract}
For $0<p<\infty$ we let $\mathcal{D}_{p-1}^{p}$ denote the space of those functions $f$ which are analytic in the unit disc $\mathbb{D}$ and satisfy $\int_{\mathbb{D}}(1-|z|)^{p-1}\left|f^{\prime}(z)\right|^{p} d A(z)<\infty$.

It is known that, whenever $p \neq q$, the only multiplier from $\mathcal{D}_{p-1}^{p}$ to $\mathcal{D}_{q-1}^{q}$ is the trivial one. However, if $X$ is a subspace of the Bloch space and $0<p \leq q<\infty$, then $X \cap \mathcal{D}_{p-1}^{p} \subset X \cap \mathcal{D}_{q-1}^{q}$, a fact which implies that the space of multipliers $M\left(\mathcal{D}_{p-1}^{p} \cap X, \mathcal{D}_{q-1}^{q} \cap X\right)$ is non-trivial.

In this paper we study the spaces of multipliers $M\left(\mathcal{D}_{p-1}^{p} \cap X, \mathcal{D}_{q-1}^{q} \cap X\right)(0<$ $p, q<\infty)$ for distinct classical subspaces $X$ of the Bloch space. Specifically, we shall take $X$ to be $H^{\infty}, B M O A$ and the Bloch space $\mathcal{B}$.
\end{abstract}

\section{INTRODUCTION AND MAIN RESULTS}

Let $\mathbb{D}=\{z \in \mathbb{C}:|z|<1\}$ denote the open unit disc in the complex plane $\mathbb{C}$ and let $\mathcal{H}$ ol $(\mathbb{D})$ be the space of all analytic functions in $\mathbb{D}$ endowed with the topology of uniform convergence in compact subsets.

If $0<r<1$ and $f \in \mathcal{H o l}(\mathbb{D})$, we set

$$
\begin{gathered}
M_{p}(r, f)=\left(\frac{1}{2 \pi} \int_{0}^{2 \pi}\left|f\left(r e^{i t}\right)\right|^{p} d t\right)^{1 / p}, 0<p<\infty, \\
M_{\infty}(r, f)=\sup _{|z|=r}|f(z)| .
\end{gathered}
$$

Whenever $0<p \leq \infty$ the Hardy space $H^{p}$ consists of those $f \in \mathcal{H o l}(\mathbb{D})$ such that $\|f\|_{H^{p}} \stackrel{\text { def }}{=} \sup _{0<r<1} M_{p}(r, f)<\infty$ (see [9] for the theory of $H^{p}$-spaces). If $0<p<\infty$ and $\alpha>-1$, the weighted Bergman space $A_{\alpha}^{p}$ consists of those $f \in \mathcal{H}$ ol $(\mathbb{D})$ such that

$$
\|f\|_{A_{\alpha}^{p}} \stackrel{\text { def }}{=}\left((\alpha+1) \int_{\mathbb{D}}(1-|z|)^{\alpha}|f(z)|^{p} d A(z)\right)^{1 / p}<\infty .
$$

The unweighted Bergman space $A_{0}^{p}$ is simply denoted by $A^{p}$. Here, $d A(z)=\frac{1}{\pi} d x d y$ denotes the normalized Lebesgue area measure in $\mathbb{D}$. We refer to [11, 23] and [40] for the theory of these spaces.

The space $\mathcal{D}_{\alpha}^{p}(0<p<\infty, \alpha>-1)$ consists of those $f \in \mathcal{H}$ ol $(\mathbb{D})$ such that $f^{\prime} \in A_{\alpha}^{p}$. Hence, if $f$ is analytic in $\mathbb{D}$, then $f \in \mathcal{D}_{\alpha}^{p}$ if and only if

$$
\|f\|_{\mathcal{D}_{\alpha}^{p}}^{p} \stackrel{\text { def }}{=}|f(0)|^{p}+\left\|f^{\prime}\right\|_{A_{\alpha}^{p}}^{p}<\infty .
$$

Date: November 20, 2012.

2010 Mathematics Subject Classification. Primary 47B35; Secondary 30H10.

Key words and phrases. Bloch space, BMOA, Hardy spaces, Spaces of Dirichlet type, Multipliers, Lacunary power series, Random power series.

This research is supported by a grant from la Dirección General de Investigación, Spain (MTM201125502) and by a grant from la Junta de Andalucía (P09-FQM-4468 and FQM-210). The third author is supported also by the "Ramón y Cajal program", 
If $p<\alpha+1$ then it is well known that $\mathcal{D}_{\alpha}^{p}=A_{\alpha-p}^{p}$ (see, e.g. Theorem 6 of [12]). On the other hand, if $p>\alpha+2$ then $\mathcal{D}_{\alpha}^{p} \subset H^{\infty}$. Therefore $\mathcal{D}_{\alpha}^{p}$ becomes a "proper Dirichlet space" when $\alpha+1 \leq p \leq \alpha+2$. The spaces $\mathcal{D}_{p-1}^{p}$ are closely related with Hardy spaces. Indeed, it is well known that $D_{1}^{2}=H^{2}$. We have also [26]

$$
H^{p} \subsetneq \mathcal{D}_{p-1}^{p}, \quad \text { for } 2 \leq p<\infty
$$

and $[12,36]$

$$
\mathcal{D}_{p-1}^{p} \subsetneq H^{p}, \quad \text { for } 0<p \leq 2 .
$$

We remark that for $p \neq q$ there is no relation of inclusion between $\mathcal{D}_{p-1}^{p}$ and $\mathcal{D}_{q-1}^{q}$ (see, e. g., [5] and [19]).

We recall that the Bloch space $\mathcal{B}$ consists of those $f \in \mathcal{H o l}(\mathbb{D})$ such that

$$
\|f\|_{\mathcal{B}}=|f(0)|+\sup _{z \in \mathbb{D}}\left(1-|z|^{2}\right)\left|f^{\prime}(z)\right|<\infty .
$$

We refer to 2 for the theory of Bloch functions.

Next, we consider multiplication operators. For $g \in \mathcal{H}$ ol $(\mathbb{D})$, the multiplication operator $M_{g}$ is defined by

$$
M_{g}(f)(z) \stackrel{\text { def }}{=} g(z) f(z), \quad f \in \mathcal{H} \operatorname{lol}(\mathbb{D}), z \in \mathbb{D} .
$$

If $X$ and $Y$ are two normed (or Fréchet) spaces of analytic functions in $\mathbb{D}$ which are continuously contained in $\mathcal{H} o l(\mathbb{D}), M(X, Y)$ will denote the space of multipliers from $X$ to $Y$,

$$
M(X, Y)=\{g \in \mathcal{H} \text { ol }(\mathbb{D}): f g \in Y, \quad \text { for all } f \in X\}
$$

and $\left\|M_{g}\right\|_{(X \rightarrow Y)}$ will denote the norm of the operator $M_{g}$. If $X=Y$ we simply write $M(X)$. These operators have been studied on the Dirichlet type spaces $\mathcal{D}_{\alpha}^{p}$ in $[20,21,15$, where among other results it is proved that

$$
M\left(\mathcal{D}_{p-1}^{p}, \mathcal{D}_{q-1}^{q}\right)=\{0\}, \quad 0<p, q<\infty, p \neq q .
$$

The following simple observation plays an important role in the motivation of this work.

Lemma 1. Suppose that $0<p<q<\infty$ and $f \in \mathcal{D}_{p-1}^{p} \cap \mathcal{B}$. Then $f \in \mathcal{D}_{q-1}^{q}$.

Proof. Since $f \in \mathcal{B}$ we have that $\sup _{z \in \mathbb{D}}(1-|z|)\left|f^{\prime}(z)\right|=M<\infty$. Using this we obtain

$$
\begin{aligned}
\int_{\mathbb{D}}(1-|z|)^{q-1}\left|f^{\prime}(z)\right|^{q} d A(z)= & \int_{\mathbb{D}}\left[(1-|z|)\left|f^{\prime}(z)\right|\right]^{q-p}(1-|z|)^{p-1}\left|f^{\prime}(z)\right|^{p} d A(z) \\
& \leq M^{q-p} \int_{\mathbb{D}}(1-|z|)^{p-1}\left|f^{\prime}(z)\right|^{p} d A(z)<\infty .
\end{aligned}
$$

Hence, $f \in \mathcal{D}_{q-1}^{q}$.

Consequently, we have:

If $X$ is a subspace of the Bloch space then

$$
X \cap \mathcal{D}_{p-1}^{p} \subset X \cap \mathcal{D}_{q-1}^{q}, \quad \text { if } 0<p \leq q<\infty,
$$

a fact which, contrary to (1.3), implies that whenever $0<p \leq q<\infty$, the space of multipliers $M\left(\mathcal{D}_{p-1}^{p} \cap X, \mathcal{D}_{q-1}^{q} \cap X\right)$ is non-trivial.

If $X \subset \mathcal{B}$, the space $X \cap \mathcal{D}_{p-1}^{p}$ is equipped with the norm

$$
\|f\|_{X \cap \mathcal{D}_{p-1}^{p}} \stackrel{\text { def }}{=}\|f\|_{X}+\|f\|_{\mathcal{D}_{p-1}^{p}} .
$$


Our aim is this paper is to obtain a characterization of the spaces $M\left(\mathcal{D}_{p-1}^{p} \cap X, \mathcal{D}_{q-1}^{q} \cap X\right)$ $(0<p, q<\infty)$ for some important subspaces $X$ of the Bloch space.

Let us start with $X=\mathcal{B}$. For $\alpha>0$, the $\alpha$-logarithmic-Bloch space $\mathcal{B}_{\log , \alpha}$ consists of those $g \in \mathcal{H}$ ol $(\mathbb{D})$ such that

$$
\rho_{\alpha}(f) \stackrel{\text { def }}{=} \sup _{z \in \mathbb{D}}\left(1-|z|^{2}\right)\left|g^{\prime}(z)\right|\left(\log \frac{e}{1-|z|^{2}}\right)^{\alpha}<\infty .
$$

It is clear that

$$
\mathcal{B}_{\log , \alpha} \subset \mathcal{B}_{\log , \beta}, \quad \alpha \geq \beta .
$$

For simplicity, the space $\mathcal{B}_{\log , 1}$ will be denoted by $\mathcal{B}_{\log }$.

The multipliers of the Bloch space into itself were characterized independently by several authors (see [3, 6, 39]). Namely, we have the following result:

$$
M(\mathcal{B})=\mathcal{B}_{\log } \cap H^{\infty} .
$$

Let us turn our attention to the spaces $M\left(\mathcal{D}_{p-1}^{p} \cap \mathcal{B}, \mathcal{D}_{q-1}^{q} \cap \mathcal{B}\right)$. Among other results, we shall prove that, for $p>1$, the space $M\left(\mathcal{B} \cap \mathcal{D}_{p-1}^{p}\right)$ coincides $M(\mathcal{B})$. This is part of the following result.

Theorem 1. Let $0<p, q<\infty$ and $g \in \mathcal{H o l}(\mathbb{D})$.

(i) If $1<q$ and $0<p \leq q<\infty$, then,

$$
M\left(\mathcal{B} \cap \mathcal{D}_{p-1}^{p}, \mathcal{B} \cap \mathcal{D}_{q-1}^{q}\right)=M(\mathcal{B}) .
$$

(ii) If $0<q<p<\infty$, then

$$
M\left(\mathcal{B} \cap \mathcal{D}_{p-1}^{p}, \mathcal{B} \cap \mathcal{D}_{q-1}^{q}\right)=\{0\} .
$$

The question of obtaining a complete characterization of $M\left(\mathcal{B} \cap \mathcal{D}_{p-1}^{p}, \mathcal{B} \cap \mathcal{D}_{q-1}^{q}\right)$ in the case $0<p \leq q \leq 1$ remains open. However, we remark that the inclusion

$$
M\left(\mathcal{B} \cap \mathcal{D}_{p-1}^{p}, \mathcal{B} \cap \mathcal{D}_{q-1}^{q}\right) \subset M(\mathcal{B}),
$$

is true for any $p, q$ (see the proof of Theorem 11 in Section [3). Using this, the fact that $M\left(\mathcal{B} \cap \mathcal{D}_{p-1}^{p}, \mathcal{B} \cap \mathcal{D}_{q-1}^{q}\right) \subset \mathcal{B} \cap \mathcal{D}_{q-1}^{q}$, and the following result we see that part (i) of Theorem 1 does not remain true for $0<q \leq 1$.

Theorem 2. If $0<q \leq 1$, then $M(\mathcal{B}) \backslash \mathcal{D}_{q-1}^{q} \neq\{0\}$.

Let us now consider the spaces $M\left(H^{\infty} \cap \mathcal{D}_{p-1}^{p}, H^{\infty} \cap \mathcal{D}_{q-1}^{q}\right)$. It is easy to prove the following result for the case $p \leq q$.

Theorem 3. If $0<p \leq q<\infty$, then $M\left(H^{\infty} \cap \mathcal{D}_{p-1}^{p}, H^{\infty} \cap \mathcal{D}_{q-1}^{q}\right)=H^{\infty} \cap \mathcal{D}_{q-1}^{q}$.

Regarding the case $0<q<p$, let us notice that if $2 \leq q<p$ then $H^{\infty} \cap \mathcal{D}_{p-1}^{p}=$ $H^{\infty} \cap \mathcal{D}_{q-1}^{q}=H^{\infty}$. Hence we have

$$
M\left(H^{\infty} \cap \mathcal{D}_{p-1}^{p}, H^{\infty} \cap \mathcal{D}_{q-1}^{q}\right)=H^{\infty}, \quad 2 \leq q<p
$$

When $0<q<p$ and $0<q<2$ the question is more complicated. It is well known (see [17, Theorem 1] and [36]) that, whenever $0<q<2$, there exists a function $f \in H^{\infty} \backslash \mathcal{D}_{q-1}^{q}$. We improve this result in our next theorem. 
Theorem 4. If $0<q<\min \{p, 2\}$, then there exists a function $f \in\left(H^{\infty} \cap \mathcal{D}_{p-1}^{p}\right) \backslash$ $\left(H^{\infty} \cap \mathcal{D}_{q-1}^{q}\right)$.

The functions constructed in Theorem 4 are used in a basic way in the proof of part (a) of our following result.

\section{Theorem 5.}

(a) If $0<q<1$ and $0<q<p<\infty$ then $M\left(H^{\infty} \cap \mathcal{D}_{p-1}^{p}, H^{\infty} \cap \mathcal{D}_{q-1}^{q}\right)=\{0\}$.

(b) If $1 \leq q<2 \leq p$ then $M\left(H^{\infty} \cap \mathcal{D}_{p-1}^{p}, H^{\infty} \cap \mathcal{D}_{q-1}^{q}\right)=\{0\}$.

In order to prove part $(b)$, we use strongly [17, Theorem 1] which asserts that, whenever $0<q<2$, there exists a function $f \in H^{\infty}$ such that

$$
\int_{0}^{1}(1-r)^{q-1}\left|f^{\prime}\left(r e^{i \theta}\right)\right|^{q} d r=\infty, \quad \text { for almost every } \theta \in \mathbb{R} .
$$

The case $1 \leq q<p<2$ of Theorem 5 remains open. However, if the answer to the following open question were affirmative then it would follow that the space $M\left(H^{\infty} \cap\right.$ $\mathcal{D}_{p-1}^{p}, H^{\infty} \cap \mathcal{D}_{q-1}^{q}$ ) would be trivial also for this range of parameters. (See the proof of Theorem $5(\mathrm{~b}))$.

Question 1. Suppose that $0<q<p<2$. Does there exist a function $f \in H^{\infty} \cap \mathcal{D}_{p-1}^{p}$ satisfying (1.8)?

We end up taking $X=B M O A$, the space of those functions $f \in H^{1}$ whose boundary values have bounded mean oscillation on the unit circle $\partial \mathbb{D}$ as defined by John and Nirenberg 24]. A lot of information about the space $B M O A$ and can be found in [4, 16, 18. Let us recall here that

$$
H^{\infty} \subsetneq B M O A \subsetneq \mathcal{B}, \quad \text { and } \quad H^{\infty} \subsetneq B M O A \subsetneq \cap_{0<p<\infty} H^{p} .
$$

We emphasize also that $B M O A$ can be characterized in terms of Carleson measures. If $I \subset \partial \mathbb{D}$ is an interval, $|I|$ will denote the length of $I$. The Carleson box $S(I)$ is defined as $S(I)=\left\{r e^{i t}: e^{i t} \in I, \quad 1-\frac{|I|}{2 \pi} \leq r<1\right\}$. If $\mu$ is a positive Borel measure in $\mathbb{D}$, we shall say that $\mu$ is a Carleson measure if there exists a positive constant $C$ such that

$$
\mu(S(I)) \leq C|I|, \quad \text { for any interval } I \subset \partial \mathbb{D} .
$$

We have (see, e.g. [18, Theorem 6.5]):

A function $f \in \mathcal{H o l}(\mathbb{D})$ belongs to $B M O A$ if and only if the Borel measure $\mu_{f}$ in $\mathbb{D}$ defined by $d \mu_{f}(z)=\left(1-|z|^{2}\right)\left|f^{\prime}(z)\right|^{2} d A(z)$ is a Carleson measure.

The multipliers of the space BMOA have been characterized in [28] (see also [34] and [38]). Indeed, we have

$$
M(B M O A)=H^{\infty} \cap B M O A_{\log } .
$$

Here, $B M O A_{\log }$ is the space of those functions $g \in H^{1}$ for which there exists a positive constant $C$ such that

$$
\int_{S(I)}\left(1-|z|^{2}\right)\left|g^{\prime}(z)\right|^{2} d A(z) \leq C|I|\left(\log \frac{2}{|I|}\right)^{-2}, \quad \text { for any interval } I \subset \partial \mathbb{D} .
$$

Let us mention that $B M O A_{\log }$ is called $L M O A$ in 34. Following the terminology of 38, we have: 
$B M O A_{\log }$ is the space of those functions $g \in H^{1}$ for which the Borel measure $\mu_{g}$ in $\mathbb{D}$ defined by $d \mu_{g}(z)=\left(1-|z|^{2}\right)\left|g^{\prime}(z)\right|^{2} d A(z)$ is a 2-logarithmic Carleson measure.

In order to make a proper study of the spaces of multipliers $M\left(\mathcal{D}_{p-1}^{p} \cap B M O A, \mathcal{D}_{q-1}^{q} \cap\right.$ $B M O A$ ), we shall present in sections 5 and 6 a series of results concerning the space $B M O A_{\log }$, some of which are of independent interest.

In section 5 we shall prove directly that $B M O A_{\log } \subsetneq \mathcal{B}_{\log } \subsetneq B M O A$ and we shall also find some simple conditions on a function $f \in \mathcal{H}$ ol $(\mathbb{D})$ which implies its membership to $B M O A_{\log }$. As a corollary we shall prove the following result about lacunary power series in $B M O A_{\log }$.

Proposition 1. Let $f \in \mathcal{H}$ ol $(\mathbb{D})$ be given by a lacunary power series, i.,e., $f$ is of the form

$$
f(z)=\sum_{k=0}^{\infty} a_{k} z^{n_{k}} \quad(z \in \mathbb{D}) \text { with } n_{k+1} \geq \lambda n_{k} \text { for all } k \text {, for a certain } \lambda>1 .
$$

If $\sum_{k=0}^{\infty}\left|a_{k}\right|^{2}\left(\log n_{k}\right)^{3}<\infty$, then $f \in B M O A_{\log } \cap H^{\infty}$.

Section [ deals with random power series of the form

$$
f_{t}(z)=\sum_{n=0}^{\infty} r_{n}(t) a_{n} z^{n}, \quad z \in \mathbb{D}, \quad 0 \leq t \leq 1,
$$

where $f(z)=\sum_{n=0}^{\infty} a_{n} z^{n}$ is analytic in $\mathbb{D}$ and $\left\{r_{n}\right\}_{n=0}^{\infty}$ is the sequence of Rademacher function (see Section 2). Among other results, we establish a sharp condition on the Taylor coefficients $a_{n}$ of $f$ which implies the almost sure membership of $f_{t}$ in $B M O A_{\log }$.

Theorem 6. function

(i) If $\sum_{n=1}^{\infty}\left|a_{n}\right|^{2}(\log n)^{3}<\infty$ then for almost every $t \in[0,1]$, the

$$
f_{t}(z)=\sum_{n=1}^{\infty} r_{n}(t) a_{n} z^{n}, \quad z \in \mathbb{D}
$$

belongs to $B M O A_{\log } \cap H^{\infty}$.

(ii) Furthermore, (i) is sharp in a very strong sense: Given a decreasing sequence of positive numbers $\left\{\delta_{n}\right\}_{n=1}^{\infty}$ with $\delta_{n} \rightarrow 0$, as $n \rightarrow \infty$, there exists a sequence of positive numbers $\left\{a_{n}\right\}_{n=1}^{\infty}$ with $\sum_{n=1}^{\infty} a_{n}^{2} \delta_{n}(\log n)^{3}<\infty$ such that, for almost every $t$ the function $f_{t}$ defined by $f_{t}(z)=\sum_{n=1}^{\infty} r_{n}(t) a_{n} z^{n}(z \in \mathbb{D})$ does not belong to $\mathcal{B}_{\log }$.

Now we pass properly to study the multipliers from $\mathcal{D}_{p-1}^{p} \cap B M O A$ to $\mathcal{D}_{q-1}^{q} \cap B M O A$ $(0<p, q<\infty)$.

If $\lambda \geq 2$ then $B M O A \subset \mathcal{D}_{\lambda-1}^{\lambda}$. Hence, trivially, we have

$$
M\left(\mathcal{D}_{p-1}^{p} \cap B M O A, \mathcal{D}_{q-1}^{q} \cap B M O A\right)=M(B M O A)=B M O A_{\log } \cap H^{\infty}, \quad 2 \leq p, q<\infty .
$$

This remains true for other values of $p$ and $q$.

Theorem 7. If $1<q<\infty$ and $0<p \leq q<\infty$, then

$$
M\left(\mathcal{D}_{p-1}^{p} \cap B M O A, \mathcal{D}_{q-1}^{q} \cap B M O A\right)=M(B M O A)=B M O A_{\log } \cap H^{\infty} .
$$

When $q<p$ then 0 is the only multiplier from $\mathcal{D}_{p-1}^{p} \cap B M O A$ to $\mathcal{D}_{q-1}^{q} \cap B M O A$, except in the cases covered by (1.10). 
Theorem 8. If $0<q<p<\infty$ and $q<2$, then

$$
M\left(\mathcal{D}_{p-1}^{p} \cap B M O A, \mathcal{D}_{q-1}^{q} \cap B M O A\right)=\{0\} .
$$

To deal with the remaining case, $0<p \leq q \leq 1$, we shall use the above mentioned results about lacunary power series and random power series. Our main results concerning random power series and multipliers are contained in the following theorem.

Theorem 9. Let $\left\{a_{n}\right\}_{n=0}^{\infty}$ be a sequence of complex numbers satisfying

$$
\sum_{n=1}^{\infty}\left|a_{n}\right|^{2}(\log n)^{3}<\infty .
$$

For $t \in[0,1]$ we set

$$
f_{t}(z)=\sum_{n=0}^{\infty} r_{n}(t) a_{n} z^{n}, \quad z \in \mathbb{D},
$$

where the $r_{n}{ }^{\prime} s$ are the Rademacher functions. Then, for almost every $t \in[0,1]$, the function $f_{t}$ satisfies the following conditions:

(i) $\int_{0}^{1}(1-r)\left(\log \frac{1}{1-r}\right)^{2}\left[M_{\infty}\left(r, f_{t}^{\prime}\right)\right]^{2} d r<\infty$.

(ii) $f_{t} \in B M O A_{\log } \cap H^{\infty}$.

(iii) $f_{t} \in M\left(\mathcal{D}_{p-1}^{p} \cap B M O A, \mathcal{D}_{q-1}^{q} \cap B M O A\right)$ whenever $0<p \leq q$ and $q>\frac{1}{2}$.

Furthermore, if $0<q<\frac{1}{2}$ then there exists a sequence $\left\{a_{n}\right\}$ which satisfies (1.11) and such that $f_{t} \notin \mathcal{D}_{q-1}^{q}$, for almost every $t$. Thus, for this sequence $\left\{a_{n}\right\}$ and for almost every $t$ we have:

(a) $f_{t} \in M(B M O A)$.

(b) If $0<p \leq \lambda$ and $\lambda>\frac{1}{2}$ then $f_{t} \in M\left(\mathcal{D}_{p-1}^{p} \cap B M O A, \mathcal{D}_{\lambda-1}^{\lambda} \cap B M O A\right)$.

(c) $f_{t} \notin M\left(\mathcal{D}_{p-1}^{p} \cap B M O A, \mathcal{D}_{q-1}^{q} \cap B M O A\right)$ whenever $0<p \leq q$.

We remark that Theorem 9 shows that Theorem[7 does not remain true for $q<1 / 2$.

Finally, we turn to consider multipliers in $M\left(\mathcal{D}_{p-1}^{p} \cap B M O A, \mathcal{D}_{q-1}^{q} \cap B M O A\right)$ given by power series with Hadamard gaps. We will show that whenever $0<p \leq q \leq 1$ the power series with Hadamard gaps in $M\left(\mathcal{D}_{p-1}^{p} \cap B M O A, \mathcal{D}_{q-1}^{q} \cap B M O A\right)$ coincide with those in $\mathcal{D}_{q-1}^{q} \cap B M O A_{\log }$ and will obtain also the analogue of Theorem 9 for lacunary power series in Theorem 14. This will give another proof of the impossibility of extending Theorem 7 to $q<1 / 2$.

\section{Preliminary Results}

As usual, a sequence of positive integers $\left\{n_{k}\right\}_{k=0}^{\infty}$ is said to be lacunar if there exists $\lambda>1$ such that $n_{k+1} \geq \lambda n_{k}$, for all $k$. Also, by a lacunary power series (also called power series with Hadamard gaps) we mean a power series of the form

$$
f(z)=\sum_{k=0}^{\infty} a_{k} z^{n_{k}}(z \in \mathbb{D}) \text { with } n_{k+1} \geq \lambda n_{k} \text { for all } k, \text { for a certain } \lambda>1 .
$$

For simplicity, we shall let $\mathcal{L}$ denote the class of all function $f \in \mathcal{H}$ ol $(\mathbb{D})$ which are given by a lacunary power series. Several known results on power series with Hadamard gaps will be repeatedly used along the paper, we collect them in the following statement, (see $[7,41,2])$. 
Proposition A. Suppose that $0<p<\infty, \alpha>-1$ and $f$ is an analytic function in $\mathbb{D}$ which is given by a power series with Hadamard gaps,

$$
f(z)=\sum_{k=0}^{\infty} a_{k} z^{n_{k}} \quad(z \in \mathbb{D}) \text { with } n_{k+1} \geq \lambda n_{k} \text { for all } k(\lambda>1) .
$$

Then:

(i) $f \in \mathcal{D}_{\alpha}^{p} \Longleftrightarrow \sum_{k=0}^{\infty} n_{k}^{p-\alpha-1}\left|a_{k}\right|^{p}<\infty$, and

$$
\|f-f(0)\|_{\mathcal{D}_{\alpha}^{p}}^{p} \asymp \sum_{k=0}^{\infty} n_{k}^{p-\alpha-1}\left|a_{k}\right|^{p} .
$$

(ii) $f \in H^{\infty}$ if and only if $\sum_{k=0}^{\infty}\left|a_{k}\right|<\infty$, and

$$
\|f\|_{H \infty} \asymp \sum_{k=0}^{\infty}\left|a_{k}\right| .
$$

(iii) $f \in \mathcal{B} \Longleftrightarrow \sup _{n}\left|a_{n}\right|<\infty$, and

$$
\|f\|_{\mathcal{B}} \asymp \sup _{n}\left|a_{n}\right| .
$$

It is also well known that $\mathcal{L} \cap H^{p}=\mathcal{L} \cap H^{2}$ for any $p \in(0, \infty)$ but

$$
H^{\infty} \cap \mathcal{L} \subsetneq H^{2} \cap \mathcal{L} .
$$

In spite of this, for any given lacunary sequence of positive integers $\left\{n_{k}\right\}_{k=1}^{\infty}$ and any sequence of complex numbers $\left\{u_{k}\right\}_{k=1}^{\infty} \in \ell^{2}$, Fournier constructed in [13] a function $f(z)=\sum_{n=0}^{\infty} a_{n} z^{n} \in H^{\infty}$ with $a_{n_{k}}=u_{k}$, for all $k$. Some properties of the bounded function $f$ which were not stated in [13] will play an important role in the proof of some of our results. Due to this fact and for sake the completeness we present a complete proof of Fournier's construction pointing out some extra properties of the constructed function (for simplicity we shall restrict to sequences $\left\{n_{k}\right\}$ satisfying $n_{k+1} \geq 2 n_{k}$ ).

Let start fixing some notation. The unit circle $\partial \mathbb{D}$ will be denoted by $\mathbb{T}$. If $g \in L^{1}(\mathbb{T})$ its Fourier coefficients $\hat{g}(n)$ are defined by

$$
\hat{g}(n)=\frac{1}{2 \pi} \int_{-\pi}^{\pi} g\left(e^{i \theta}\right) e^{-i n \theta} d \theta, \quad n \in \mathbb{Z} .
$$

If $n_{1}<n_{2}$ are integers we shall write $\left\lfloor n_{1}, n_{2}\right\rfloor$ for the set of all integers $n$ with $n_{1} \leq$ $n \leq n_{2}$. Also, for $g(z)=\sum_{k=0}^{\infty} b_{k} z^{k} \in \mathcal{H}$ ol $(\mathbb{D})$ and $n_{2}>n_{1} \geq 0$, we set

$$
S_{n_{1}, n_{2}} g(z)=\sum_{k=n_{1}}^{n_{2}-1} b_{k} z^{k} .
$$

Proposition 2. Assume that $\left\{u_{k}\right\}_{k=0}^{\infty} \in \ell^{2}$ and let $\left\{n_{k}\right\}_{k=0}^{\infty}$ be a sequence of positive integers such that $n_{k+1}>2 n_{k}$, for all $k$. Then, there exists a function $\Psi \in \mathcal{H o l}(\mathbb{D})$ of the form

$$
\Psi(z)=\sum_{n=0}^{\infty} a_{n} z^{n}, \quad z \in \mathbb{D},
$$

with the following properties:

(i) $\Psi \in H^{\infty}$.

(ii) $a_{n_{k}}=u_{k}$, for all $k$. 
(iii) If we define $\Lambda_{0}=\left\{n_{0}\right\}$ and $\Lambda_{k}=\left\lfloor n_{k}-n_{k-1}, n_{k}\right\rfloor$ for $k>0$, we have that the sets $\Lambda_{k}$ are pairwise disjoint and satisfy $\Lambda_{k} \subset\left\lfloor n_{k-1}+1, n_{k}\right\rfloor$ for all $k \geq 1$. Furthermore, $a_{n}=0$ if $n \notin \cup_{k=0}^{\infty} \Lambda_{k}$.

(iv) There is an absolute constant $C$ such that

$$
\left\|S_{n_{k}+1, n_{k+1}+1} \Psi\right\|_{H^{\infty}} \leq C\left|u_{k}\right|, \quad \text { for all } k \text {. }
$$

Proof. The construction depends on the following equality [13, p. 402]

$$
|a+v b|^{2}+|b-\bar{v} a|=\left(1+|v|^{2}\right)\left(|a|^{2}+|b|^{2}\right), \quad a, b, v \in \mathbb{C} .
$$

Let us define inductively the following sequences of functions on $\mathbb{T}$

$$
\phi_{0}(\zeta)=u_{0} \zeta^{n_{0}}, \quad h_{0}(\zeta)=1, \quad \zeta \in \mathbb{T}
$$

and, for $k>0$,

$(2.3) \phi_{k}(\zeta)=\phi_{k-1}(\zeta)+u_{k} \zeta^{n_{k}} h_{k-1}(\zeta), \quad h_{k}(\zeta)=h_{k-1}(\zeta)-\overline{u_{k}} \zeta^{-n_{k}} \phi_{k-1}(\zeta), \quad(\zeta \in \mathbb{T})$.

Since $n_{k+1}>2 n_{k}$, it is clear that the sets $\Lambda_{k}, k=1,2, \ldots$, are disjoint and that $\Lambda_{k} \subset\left\lfloor n_{k-1}+1, n_{k}\right\rfloor$ for all $k \geq 1$.

We claim that that the sequences $\left\{\phi_{k}\right\}$ and $\left\{h_{k}\right\}$ satisfy the following properties

$$
\begin{gathered}
\widehat{\phi_{k}}(n)=0, \quad \text { whenever } k \geq 0 \text { and } n \notin \bigcup_{j=0}^{k} \Lambda_{j} \\
\widehat{h_{k}}(-n)=0, \quad \text { whenever } k \geq 0 \text { and } n \geq 1 \text { and } n \notin \bigcup_{j=1}^{k} \Lambda_{j} . \\
\widehat{\phi_{k}}(n)=\widehat{\phi_{j}}(n), \quad \text { whenever } k \geq j \text { and } n \leq n_{j}, \\
\widehat{\phi_{k}}\left(n_{j}\right)=u_{j}, \quad \text { whenever } k \geq j .
\end{gathered}
$$

It is clear that (2.4) and (2.5) hold for $k=0,1$. Arguing by induction, assume that (2.4) and (2.5) are valid for some value of $k \in \mathbb{N}$. Then,

$$
\phi_{k+1}(\zeta)=\phi_{k}(\zeta)+u_{k+1} \zeta^{n_{k+1}} h_{k}(\zeta)=\sum_{j=0}^{k} \sum_{n \in \Lambda_{j}} \widehat{\phi_{k}}(n) \zeta^{n}+f_{k}(\zeta),
$$

where $f_{k}(\zeta)=u_{k+1} \zeta^{n_{k+1}} h_{k}(\zeta)$. By the induction hypotheses $\widehat{f}_{k}(n)=0$ if $n \notin \Lambda_{k+1}$, which gives (2.4) for $k+1$. The proof of (2.5) is analogous. Now, (2.6) follows from (2.3), (2.4) and the fact that the sets $\Lambda_{k}$ are disjoint and (2.5). Using again that the sets $\Lambda_{k}$ are disjoint, (2.6), (2.3) and (2.2), we deduce (2.7).

We have that

$$
\left|\phi_{0}(\zeta)\right|^{2}+\left|h_{0}(\zeta)\right|^{2}=1+\left|u_{0}\right|^{2}
$$

so if we assume that $\left|\phi_{k}(\zeta)\right|^{2}+\left|h_{k}(\zeta)\right|^{2}=\prod_{j=0}^{k}\left(1+\left|u_{j}\right|^{2}\right)$, bearing in mind (2.1) and (2.3), it follows that

$$
\left|\phi_{k+1}(\zeta)\right|^{2}+\left|h_{k+1}(\zeta)\right|^{2}=\left(1+\left|u_{k+1}\right|^{2}\right)\left(\left|\phi_{k}(\zeta)\right|^{2}+\left|h_{k}(\zeta)\right|^{2}\right)=\prod_{j=0}^{k+1}\left(1+\left|u_{j}\right|^{2}\right),
$$


hence we have proved by induction that

$$
\left|\phi_{k}(\zeta)\right|^{2}+\left|h_{k}(\zeta)\right|^{2}=\prod_{j=0}^{k}\left(1+\left|u_{j}\right|^{2}\right), \quad \zeta \in \mathbb{T}, \quad k=0,1,2, \ldots
$$

This and the fact that $\left\{u_{k}\right\}_{k=0}^{\infty} \in \ell^{2}$ imply that $\left\{h_{k}\right\}_{k=0}^{\infty}$ and $\left\{\phi_{k}\right\}_{k=0}^{\infty}$ are uniformly bounded sequences of functions in $L^{\infty}(\mathbb{T})$. Then, using the Banach-Alaoglu theorem, (2.4), (2.6) and (2.7), we deduce that a subsequence of $\left\{\phi_{k}\right\}$ converges in the weak star topology of $L^{\infty}(\mathbb{T})$ to a function $\phi \in L^{\infty}(\mathbb{T})$ with $\hat{\phi}(n)=0$ for all $n<0$, and $\hat{\phi}\left(n_{k}\right)=u_{k}$ for all $k$. Then if we set $a_{n}=\hat{\phi}(n)(n \geq 0)$ it follows that the function $\Psi$ defined by

$$
\Psi(z)=\sum_{n=0}^{\infty} a_{n} z^{n}, \quad z \in \mathbb{D},
$$

is analytic in $\mathbb{D}$ and satisfies (i), (ii) and (iii).

Finally, we shall prove (iv). Using(2.6) and (2.8), we see that for any $\zeta \in \mathbb{T}$, we have

$$
\begin{aligned}
& S_{n_{k}+1, n_{k+1}+1} \Psi(\zeta)=\sum_{n=n_{k}+1}^{n_{k+1}} \widehat{\Psi}(n) \zeta^{n}=\sum_{m=n_{k}+1}^{n_{k+1}}\left(\lim _{m \rightarrow \infty} \widehat{\phi_{m}}(n)\right) \zeta^{n} \\
& =\sum_{n=n_{k}+1}^{n_{k+1}} \widehat{\phi_{k+1}}(n) \zeta^{n}=f_{k}(\zeta)=u_{k+1} \zeta^{n_{k+1}} h_{k}(\zeta),
\end{aligned}
$$

which, bearing in mind that $\sup _{k}\left\|h_{k}\right\|_{\infty}=C<\infty$, implies

$$
\left\|S_{n_{k}+1, n_{k+1}+1} \Psi\right\|_{H^{\infty}}=\left|u_{k+1}\right||| h_{k} \|_{L^{\infty}(\mathbb{T})} \leq C\left|u_{k+1}\right| .
$$

This finishes the proof.

Our work will also make use of the Rademacher functions $\left\{r_{n}(t)\right\}_{n=0}^{\infty}$ which are are defined by

$$
\begin{gathered}
r_{0}(t)= \begin{cases}1, & \text { if } 0<t<1 / 2 \\
-1, & \text { if } 1 / 2<t<1 \\
0, & \text { if } t=0,1 / 2,1\end{cases} \\
r_{n}(t)=r_{0}\left(2^{n} t\right), \quad n=1,2, \ldots
\end{gathered}
$$

See, e. g., [41, Chapter V, Vol. I] or [9, Appendix A] for the properties of these functions. In particular, we shall use Khinchine's inequality which we state as follows.

Proposition B (Khinchine's inequality). If $\left\{c_{k}\right\}_{k=1}^{\infty} \in \ell^{2}$ then the series $\sum_{k=1}^{\infty} c_{k} r_{k}(t)$ converges almost everywhere. Furthermore, for $0<p<\infty$ there exist positive constants $A_{p}, B_{p}$ such that for every sequence $\left\{c_{k}\right\}_{k=0}^{\infty} \in \ell^{2}$ we have

$$
A_{p}\left(\sum_{k=0}^{\infty}\left|c_{k}\right|^{2}\right)^{p / 2} \leq \int_{0}^{1}\left|\sum_{k=0}^{\infty} c_{k} r_{k}(t)\right|^{p} d t \leq B_{p}\left(\sum_{k=0}^{\infty}\left|c_{k}\right|^{2}\right)^{p / 2}
$$




\section{Multipliers on $\mathcal{B} \cap \mathcal{D}_{p-1}^{p}$}

Proof of Theorem 1. (i) Assume that $g \in M\left(\mathcal{B} \cap \mathcal{D}_{p-1}^{p}, \mathcal{B} \cap \mathcal{D}_{q-1}^{q}\right)$. From now and throughout the paper we shall denote by $\varphi_{a}$ the Möbius transformation which interchanges the origin and $a$,

$$
\varphi_{a}(z)=\frac{a-z}{1-\bar{a} z}, \quad z \in \mathbb{D} .
$$

A simple calculation shows that

$$
\sup _{a \in \mathbb{D}}\left\|\varphi_{a}\right\|_{{\mathcal{B} \cap \mathcal{D}_{p-1}^{p}}_{p}<\infty .}
$$

So, for any $a, z \in \mathbb{D}$

$$
\begin{aligned}
& \left(1-|z|^{2}\right)\left|\varphi_{a}^{\prime}(z) g(z)\right|=\left(1-|z|^{2}\right)\left|\left(\varphi_{a} \cdot g\right)^{\prime}(z)-\varphi_{a}(z) g^{\prime}(z)\right| \\
& \leq\left\|\varphi_{a} g\right\|_{\mathcal{B} \cap \mathcal{D}_{q-1}^{q}}+\left(1-|z|^{2}\right)\left|\varphi_{a}(z) g^{\prime}(z)\right| \lesssim\left\|M_{g}\right\|_{\left({\left.\mathcal{B} \cap \mathcal{D}_{p-1}^{p} \rightarrow \mathcal{B}^{p} \mathcal{D}_{q-1}^{q}\right)}^{q}+\|g\|_{\mathcal{B}}<\infty .\right.}
\end{aligned}
$$

Since $\left(1-|a|^{2}\right)\left|\varphi_{a}^{\prime}(a)\right|=1$, taking $z=a$ in (3.1) we obtain

$$
|g(a)| \lesssim\left\|M_{g}\right\|_{\left(\mathcal{B} \cap \mathcal{D}_{p-1}^{p} \rightarrow{\left.\mathcal{B} \cap \mathcal{D}_{q-1}^{q}\right)}^{q}\right.}+\|g\|_{\mathcal{B}}<\infty
$$

for any $a \in \mathbb{D}$. Thus, $g \in H^{\infty}$.

Next consider the family of test functions, $f_{\theta}(z)=\log \frac{1}{1-z e^{-i \theta}}, \theta \in[0,2 \pi)$. A calculation shows that $\left\{f_{\theta}\right\}_{\theta \in[0,2 \pi)}$ is uniformly bounded in $\mathcal{B} \cap \mathcal{D}_{p-1}^{p}$. Therefore,

$$
\begin{aligned}
A & =\sup _{\theta \in[0,2 \pi)}\left\|g f_{\theta}\right\|_{\mathcal{B}} \leq \sup _{\theta \in[0,2 \pi)}\left\|g f_{\theta}\right\|_{\mathcal{B}_{\mathcal{B}} \mathcal{D}_{q-1}^{q}} \\
& \leq\left\|M_{g}\right\|_{\left(\mathcal{B} \cap \mathcal{D}_{p-1}^{p} \rightarrow \mathcal{B} \cap \mathcal{D}_{q-1}^{q}\right)} \sup _{\theta \in[0,2 \pi)}\left\|f_{\theta}\right\|_{\mathcal{B} \cap \mathcal{D}_{p-1}^{p}}<\infty,
\end{aligned}
$$

which implies that

$$
\begin{aligned}
\left(1-|z|^{2}\right)\left|g^{\prime}(z) f_{\theta}(z)\right| & =\left(1-|z|^{2}\right)\left|g^{\prime}(z) f_{\theta}(z)+g(z) f_{\theta}^{\prime}(z)-g(z) f_{\theta}^{\prime}(z)\right| \\
& \leq A+\left(1-|z|^{2}\right)\left|g(z) f_{\theta}^{\prime}(z)\right| \\
& =A+\|g\|_{H^{\infty}} \sup _{\theta \in[0,2 \pi)}\left\|f_{\theta}\right\|_{\mathcal{B}} \\
& <\infty, \quad \text { for all } z \in \mathbb{D} \text { and } \theta \in[0,2 \pi) .
\end{aligned}
$$

Finally, given $z \in \mathbb{D}$ choose $e^{i \theta}=\frac{z}{|z|}$ to deduce that

$$
\sup _{z \in \mathbb{D}}\left|g^{\prime}(z)\right|(1-|z|) \log \frac{1}{1-|z|}<\infty,
$$

which together the fact that $g \in H^{\infty}$ gives that $g \in M(\mathcal{B})$.

Suppose now that $g \in M(\mathcal{B})$ and take $f \in \mathcal{B} \cap \mathcal{D}_{p-1}^{p}$. Then $f g \in \mathcal{B}$. Using Lemma 1 and the closed graph theorem, we obtain

$$
\begin{aligned}
& \int_{\mathbb{D}}\left|(f g)^{\prime}(z)\right|^{q}\left(1-|z|^{2}\right)^{q-1} d A(z) \\
& \lesssim \int_{\mathbb{D}}\left|f^{\prime}(z) g(z)\right|^{q}\left(1-|z|^{2}\right)^{q-1} d A(z)+\int_{\mathbb{D}}\left|g^{\prime}(z) f(z)\right|^{q}\left(1-|z|^{2}\right)^{q-1} d A(z) \\
& \lesssim\|g\|_{H^{\infty}}^{q}\|f\|_{\mathcal{B} \cap \mathcal{D}_{p-1}^{p}}^{q}+\int_{\mathbb{D}}\left|f(z) g^{\prime}(z)\right|^{q}\left(1-|z|^{2}\right)^{q-1} d A(z) .
\end{aligned}
$$


We shall distinguish two cases to deal with the last integral which appears in (3.2). First, if $1<q \leq 2$, bearing in mind (1.2) and the fact that $g \in \mathcal{B}_{\log }$, we see that

$$
\begin{aligned}
\int_{\mathbb{D}}\left|f(z) g^{\prime}(z)\right|^{q}\left(1-|z|^{2}\right)^{q-1} d A(z) & \lesssim \int_{0}^{1} \frac{1}{(1-r)^{q} \log ^{q} \frac{e}{1-r}} M_{q}^{q}(r, f)(1-r)^{q-1} d r \\
& \lesssim\|f\|_{\mathcal{D}_{q-1}^{q}}^{q} \int_{0}^{1} \frac{1}{(1-r) \log ^{q} \frac{e}{1-r}} d r \\
& \lesssim\|f\|_{\mathcal{B}_{\mathcal{B}} \mathcal{D}_{p-1}^{p}}
\end{aligned}
$$

On the other hand, if $2<q<\infty$, then using that $g \in \mathcal{B}_{\log }$ and the well known fact that

$$
M_{q}(r, f) \leq C\|f\|_{\mathcal{B}}\left(\log \frac{1}{1-r}\right)^{1 / 2}, \quad 0<r<1,
$$

(see, e.g., [8]) we get

$$
\begin{aligned}
\int_{\mathbb{D}}\left|f(z) g^{\prime}(z)\right|^{q}\left(1-|z|^{2}\right)^{q-1} d A(z) & \lesssim \int_{0}^{1} \frac{1}{(1-r)^{q} \log ^{q} \frac{e}{1-r}} M_{q}^{q}(r, f)(1-r)^{q-1} d r \\
& \lesssim\|f\|_{\mathcal{B}}^{q} \int_{0}^{1} \frac{1}{(1-r) \log ^{q / 2} \frac{e}{1-r}} d r<\infty .
\end{aligned}
$$

Joining (3.2) (3.3) and (3.4), we see that in any case we have $f g \in \mathcal{D}_{q-1}^{q}$ and, hence, $f g \in \mathcal{B} \cap \mathcal{D}_{q-1}^{q}$. Thus, we have proved that $g \in M\left(\mathcal{B} \cap \mathcal{D}_{p-1}^{p}, \mathcal{B} \cap \mathcal{D}_{q-1}^{q}\right)$ finishing the proof.

(ii) We borrow ideas from [15, Theorem 12]. We shall distinguish three cases.

Case 1. $\mathbf{2}<\mathbf{q}<\infty$. Assume that $g \in M\left(\mathcal{B} \cap \mathcal{D}_{p-1}^{p}, \mathcal{B} \cap \mathcal{D}_{q-1}^{q}\right)$ and $g \not \equiv 0$. By the proof of [15, Theorem K] (see also the proofs of [19, Theorems 1.6 and 1.7]), it follows that there exists a function $f \in \mathcal{D}_{p-1}^{p}$, given by a lacunary power series, with $f(0) \neq 0$, and such that its sequence of ordered zeros $\left\{z_{n}\right\}$ (that is, the $z_{n}^{\prime} s$ are ordered so that $\left.\left|z_{1}\right| \leq\left|z_{2}\right| \leq\left|z_{3}\right| \ldots\right)$ satisfies

$$
\prod_{n=1}^{N} \frac{1}{\left|z_{n}\right|} \neq o(\log N)^{\frac{1}{2}-\frac{1}{q}}
$$

Since $f$ is given by a lacunary power series, by Proposition $\mathrm{A}$, the sequence of its Taylor coefficients is in $\ell^{p}$. This implies that $f \in \mathcal{B} \cap \mathcal{D}_{p-1}^{p}$. If $\left\{w_{n}\right\}$ is the sequence of non-zero zeros of $g f$ arranged so that $\left|w_{1}\right| \leq\left|w_{2}\right| \leq\left|w_{3}\right| \ldots$, we have that $\left|w_{n}\right| \leq\left|z_{n}\right|$, for all $n$, which gives that

$$
\prod_{n=1}^{N} \frac{1}{\left|w_{n}\right|} \geq \prod_{n=1}^{N} \frac{1}{\left|z_{n}\right|}
$$

hence

$$
\prod_{n=1}^{N} \frac{1}{\left|w_{n}\right|} \neq o(\log N)^{\frac{1}{2}-\frac{1}{q}} .
$$

This together with [19, Theorem 1.6] implies that $f g \notin \mathcal{D}_{q-1}^{q}$. This is a contradiction. Thus, $g \equiv 0$. 
Case 2. $\mathbf{0}<\mathbf{q} \leq \mathbf{2}<\mathbf{p}$. The proof is similar to that of the case 1. Suppose that $g \not \equiv 0$ and $g \in M\left(\mathcal{B} \cap \mathcal{D}_{p-1}^{p}, \mathcal{B} \cap \mathcal{D}_{q-1}^{q}\right)$. Take $\gamma \in\left(0, \frac{1}{2}-\frac{1}{p}\right)$. Then, by the proof of [15. Theorem K] and Proposition $\left[\mathrm{A}\right.$, there is a function $f \in \mathcal{B} \cap \mathcal{D}_{p-1}^{p}$, represented by a lacunary series, with $f(0) \neq 0$ whose sequence of ordered zeros $\left\{z_{n}\right\}$ satisfies

$$
\prod_{n=1}^{N} \frac{1}{\left|z_{n}\right|} \neq o(\log N)^{\gamma}
$$

Let $\left\{w_{n}\right\}_{n=1}^{\infty}$ be the sequence of ordered non-zero zeros of $f g$. Since $f g \in \mathcal{D}_{q-1}^{q}$ and $q \leq 2$, it follows that $f g \in H^{q}$ and, hence, $\left\{w_{n}\right\}_{n=1}^{\infty}$ satisfies the Blaschke condition which is equivalent to saying that

$$
\prod_{k=1}^{N} \frac{1}{\left|w_{k}\right|}=\mathrm{O}(1), \quad \text { as } N \rightarrow \infty \text {. }
$$

This is in contradiction with (3.5), because any zero of $f$ is also a zero of $f g$. Consequently, $g \equiv 0$.

Case 3. $\mathbf{0}<\mathbf{p} \leq \mathbf{2}$. Suppose that $g \not \equiv 0$ and $g \in M\left(\mathcal{B} \cap \mathcal{D}_{p-1}^{p}, \mathcal{B} \cap \mathcal{D}_{q-1}^{q}\right)$. Take $a_{n}=\frac{1}{n^{1 / p+\varepsilon}}$ with $0<\varepsilon<\frac{1}{q}-\frac{1}{p}$ and $f(z)=\sum_{n=1}^{\infty} a_{n} z^{2^{n}}$. Since $\sum_{n=1}^{\infty} a_{n}^{p}<\infty$ and $\sum_{n=1}^{\infty} a_{n}^{q}=\infty$, then by Proposition $\mathrm{A}, f \in \mathcal{B} \cap \mathcal{D}_{p-1}^{p} \backslash \mathcal{D}_{q-1}^{q}$.

Let $\left\{r_{k}(t)\right\}$ be the Rademacher functions and let $f_{t}(z)=\sum_{k=1}^{\infty} r_{k}(t) a_{k} z^{2^{k}}$. By Proposition $\mathrm{A}$ (iii)

$$
\|f\|_{\mathcal{B}} \asymp \sup _{n}\left|a_{n}\right| \asymp\left\|f_{t}\right\|_{\mathcal{B}}, \quad t \in[0,1]
$$

and

$$
\left\|f_{t}\right\|_{H^{2}}^{2 p}=\left(\sum_{k=0}^{\infty}\left|a_{k}\right|^{2}\right)^{p} \leq\left(\sum_{k=0}^{\infty}\left|a_{k}\right|^{p}\right)^{2} \asymp\left\|f_{t}\right\|_{\mathcal{D}_{p-1}^{p}}^{2 p} \asymp\|f\|_{\mathcal{D}_{p-1}^{p}}^{2 p}, \quad t \in[0,1]
$$

Then for any $t \in[0,1]$, it follows that

$$
\int_{\mathbb{D}}\left|\left(g f_{t}\right)^{\prime}(z)\right|^{q}\left(1-|z|^{2}\right)^{q-1} d A(z) \lesssim\left\|f_{t}\right\|_{\mathcal{D}_{p-1}^{p}}^{q}+\left\|f_{t}\right\|_{\mathcal{B}}^{q} \asymp\|f\|_{\mathcal{D}_{p-1}^{p}}^{q}+\|f\|_{\mathcal{B}}^{q}<\infty .
$$

So, by Fubini's theorem, Khinchine's inequality and the fact that $g \in \mathcal{D}_{q-1}^{q}$, we obtain

$$
\begin{aligned}
& \int_{0}^{1} \int_{\mathbb{D}}\left|g f_{t}^{\prime}(z)\right|^{q}\left(1-|z|^{2}\right)^{q-1} d A(z) d t \\
& \lesssim \int_{0}^{1} \int_{\mathbb{D}}\left|\left(g f_{t}\right)^{\prime}(z)\right|^{q}\left(1-|z|^{2}\right)^{q-1} d A(z) d t+\int_{0}^{1} \int_{\mathbb{D}}\left|f_{t} g^{\prime}(z)\right|^{q}\left(1-|z|^{2}\right)^{q-1} d A(z) d t \\
& \lesssim\|f\|_{\mathcal{B} \cap \mathcal{D}_{p-1}^{p}}^{q}+\int_{\mathbb{D}}\left|g^{\prime}(z)\right|^{q} \int_{0}^{1}\left|f_{t}(z)\right|^{q}\left(1-|z|^{2}\right)^{q-1} d t d A(z) \\
& \lesssim\|f\|_{\mathcal{B} \cap \mathcal{D}_{p-1}^{p}}^{q}+\int_{\mathbb{D}}\left|g^{\prime}(z)\right|^{q} M_{2}^{q}(|z|, f)\left(1-|z|^{2}\right)^{q-1} d A(z) \\
& \lesssim\|f\|_{\mathcal{B} \cap \mathcal{D}_{p-1}^{p}}^{q}+\|f\|_{\mathcal{D}_{p-1}^{p}}^{q} \int_{\mathbb{D}}\left|g^{\prime}(z)\right|^{q}\left(1-|z|^{2}\right)^{q-1} d A(z) \\
& \lesssim\|f\|_{\mathcal{B} \cap \mathcal{D}_{p-1}^{p}}^{q} \cdot
\end{aligned}
$$

On the other hand, since $g \not \equiv 0$, there exists a positive constant $C$ such that $M_{q}^{q}(r, g) \geq C$, $1 / 2<r<1$. Using Fubini's theorem, Khinchine's inequality and bearing in mind that 
$f^{\prime}$ is also given by a power series with Hadamard gaps (thus $M_{2}\left(r, f^{\prime}\right) \asymp M_{q}\left(r, f^{\prime}\right)$ ) we have that

$$
\begin{aligned}
& \int_{0}^{1} \int_{\mathbb{D}}\left|g f_{t}^{\prime}(z)\right|^{q}\left(1-|z|^{2}\right)^{q-1} d A(z) d t \\
& =\int_{\mathbb{D}}|g(z)|^{q}\left(1-|z|^{2}\right)^{q-1}\left(\int_{0}^{1}\left|f_{t}^{\prime}(z)\right|^{q} d t\right) d A(z) \\
& \asymp \int_{\mathbb{D}}|g(z)|^{q}\left(1-|z|^{2}\right)^{q-1} M_{2}^{q}\left(|z|, f^{\prime}\right) d A(z) \\
& \geq C \int_{1 / 2}^{1} M_{q}^{q}(r, g) M_{q}^{q}\left(r, f^{\prime}\right)\left(1-r^{2}\right)^{q-1} d r \\
& \geq C \int_{1 / 2}^{1} M_{q}^{q}\left(r, f^{\prime}\right)\left(1-r^{2}\right)^{q-1} d r=+\infty .
\end{aligned}
$$

This is in contradiction with (3.8). It follows that $g \equiv 0$.

We remark that the argument used to prove the inclusion $M\left(\mathcal{B} \cap \mathcal{D}_{p-1}^{p}, \mathcal{B} \cap \mathcal{D}_{q-1}^{q}\right) \subset$ $M(\mathcal{B})$ in the proof of Theorem 1 (i) works for any values of $p$ and $q$, that is we have

$$
M\left(\mathcal{B} \cap \mathcal{D}_{p-1}^{p}, \mathcal{B} \cap \mathcal{D}_{q-1}^{q}\right) \subset M(\mathcal{B}), \quad 0<p, q<\infty .
$$

We do not have a complete characterization of the space $M\left(\mathcal{B} \cap \mathcal{D}_{p-1}^{p}, \mathcal{B} \cap \mathcal{D}_{q-1}^{q}\right)$ in the case $0<p \leq q \leq 1$, however we find a sharp sufficient condition on a function $g$ to lie in this space of multipliers. We note that Theorem 2 is a byproduct of part (ii) of the following stronger result.

Proposition 3. Let $0<p \leq q \leq 1, \alpha \in\left(\frac{1}{q}, \infty\right)$ and $g \in \mathcal{H}$ ol $(\mathbb{D})$. Then,

(i) If $g \in \mathcal{B}_{\log , \alpha} \cap H^{\infty}$, then $g \in M\left(\mathcal{B} \cap \mathcal{D}_{p-1}^{p}, \mathcal{B} \cap \mathcal{D}_{q-1}^{q}\right)$.

(ii) $\left(\mathcal{B}_{\log , \frac{1}{q}} \cap H^{\infty}\right) \backslash \mathcal{D}_{q-1}^{q} \neq\{0\}$.

Proof. Part (i) can be proved arguing as in (3.2) and (3.3), so we omit a detailed proof.

(ii) Assume first that $0<q<1$. Consider the lacunary power series

$$
g(z)=\sum_{k=1}^{\infty} \frac{1}{k^{1 / q}} z^{2^{k}}
$$

By Proposition A] $g \in H^{\infty} \backslash \mathcal{D}_{q-1}^{q}$. Since $\limsup _{k \rightarrow \infty} \frac{1}{k^{1 / q}}\left(\log 2^{k}\right)^{1 / q}<\infty($ see [32, p. 20]) $g \in \mathcal{B}_{\log , \frac{1}{q}}$.

Let us consider now the case $q=1$. The proof in this case is a little bit more involved. Set

$$
u_{k}=\frac{1}{k+1} \text { and } n_{k}=4^{k}, \quad k=0,1,2, \ldots
$$

Let $\Psi$ be the $H^{\infty}$-function associated to these sequences via Theorem 2 , By [36, Lemma $1.6(\mathrm{i})]$,

$$
\|\Psi\|_{\mathcal{D}_{0}^{1}} \gtrsim \|\left\{\widehat{\Psi}\left(4^{k}\right\}_{k=0}^{\infty} \|_{\ell^{1}}=\sum_{k=0}^{\infty} \frac{1}{k+1}=\infty\right.
$$




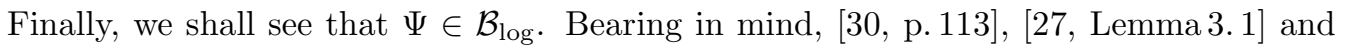
Lemma 2 (iv), we deduce

$$
\begin{aligned}
& M_{\infty}\left(r, \Psi^{\prime}\right) \leq|\widehat{\Psi}(1)|+\sum_{k=0}^{\infty} M_{\infty}\left(r, S_{n_{k}+1, n_{k+1}} \Psi^{\prime}\right) \\
& \lesssim\|\Psi\|_{H^{\infty}}+\sum_{k=0}^{\infty}\left\|S_{n_{k}+1, n_{k+1}} \Psi^{\prime}\right\|_{H^{\infty}} r^{4^{k}} \\
& \lesssim\|\Psi\|_{H^{\infty}}+\sum_{k=0}^{\infty} 4^{k}\left\|S_{n_{k}+1, n_{k+1}} \Psi\right\|_{H \infty} r^{4^{k}} \\
& \lesssim\|\Psi\|_{H^{\infty}}+\sum_{k=0}^{\infty} \frac{4^{k}}{k+1} r^{4^{k}} .
\end{aligned}
$$

Since an standard calculation shows that

$$
\sum_{k=0}^{\infty} \frac{4^{k}}{k+1} r^{4^{k}} \lesssim \frac{1}{(1-r) \log \frac{e}{1-r}}, \quad 0 \leq r<1,
$$

this finishes the proof.

Next we provide a sufficient condition, which involves Carleson measures, on a function $g$ to lie in this space of multipliers. It turns out to be also necessary if $g$ is given by a power series with Hadamard gaps.

Theorem 10. Assume that $0<p \leq q \leq 1$ and let $g$ be an analytic function in $\mathbb{D}$. Let $\mu_{g, q}$ be the Borel measure in $\mathbb{D}$ defined by $d \mu_{g, q}(z)=\left|g^{\prime}(z)\right|^{q}\left(1-|z|^{2}\right)^{q-1} d A(z)$.

(a) If $g \in H^{\infty} \cap \mathcal{B}_{\log }$ and the measure $\mu_{g, q}$ is a Carleson measure, then $g \in M(\mathcal{B} \cap$ $\left.\mathcal{D}_{p-1}^{p}, \mathcal{B} \cap \mathcal{D}_{q-1}^{q}\right)$.

(b) If $g$ is given by a power series with Hadamard gaps, then $g \in M\left(\mathcal{B} \cap \mathcal{D}_{p-1}^{p}, \mathcal{B} \cap\right.$ $\left.\mathcal{D}_{q-1}^{q}\right)$ if and only if $g \in H^{\infty} \cap \mathcal{B}_{\log }$ and the measure $\mu_{g, q}$ is a Carleson measure.

Proof. Suppose that $g \in H^{\infty} \cap \mathcal{B}_{\log }$ and the measure $\mu_{g, q}$ is a Carleson measure. Take $f \in \mathcal{B} \cap \mathcal{D}_{p-1}^{p}$.

- Using (1.6), we see that $g \in M(\mathcal{B})$ and, hence, $f g \in \mathcal{B}$.

- Using [36, Theorem 2.1] we deduce that $g \in M\left(\mathcal{D}_{p-1}^{p}\right)$ and, then it follows that $f g \in \mathcal{D}_{p-1}^{p}$.

Since $\mathcal{D}_{p-1}^{p} \cap \mathcal{B} \subset \mathcal{D}_{q-1}^{q} \cap \mathcal{B}$, we have that $f g \in \mathcal{B} \cap \mathcal{D}_{q-1}^{q}$. Thus, we have proved that $g \in M\left(\mathcal{B} \cap \mathcal{D}_{p-1}^{p}, \mathcal{B} \cap \mathcal{D}_{q-1}^{q}\right)$. This finishes the proof of part (a).

Suppose now that $g$ is given by a power series with Hadamard gaps and $g \in M(\mathcal{B} \cap$ $\left.\mathcal{D}_{p-1}^{p}, \mathcal{B} \cap \mathcal{D}_{q-1}^{q}\right)$. Then $g \in \mathcal{D}_{q-1}^{q}$. Now, using Theorem 3.2 of [20, we see that this implies that $\mu_{g, q}$ is a Carleson measure.

\section{Multipliers ON $H^{\infty} \cap \mathcal{D}_{p-1}^{p}$}

Proof of Theorem 3. Suppose that $0<p \leq q<\infty$.

If $g \in M\left(H^{\infty} \cap \mathcal{D}_{p-1}^{p}, H^{\infty} \cap \mathcal{D}_{q-1}^{q}\right)$ then, since $H^{\infty} \cap \mathcal{D}_{p-1}^{p}$ contains the constant functions, it follows trivially that $g \in H^{\infty} \cap \mathcal{D}_{q-1}^{q}$. 
On the other hand, if $g \in H^{\infty} \cap \mathcal{D}_{q-1}^{q}$ and $f \in H^{\infty} \cap \mathcal{D}_{p-1}^{p}$, it is clear that $g f \in H^{\infty}$. We also have

$$
\begin{aligned}
& \int_{\mathbb{D}}\left|\left(g^{\prime} f+g f^{\prime}\right)(z)\right|^{q}\left(1-|z|^{2}\right)^{q-1} d A(z) \\
& \lesssim \int_{\mathbb{D}}\left|\left(g^{\prime} f\right)(z)\right|^{q}\left(1-|z|^{2}\right)^{q-1} d A(z)+\int_{\mathbb{D}}\left|g(z) f^{\prime}(z)\right|^{q}\left(1-|z|^{2}\right)^{q-1} d A(z) \\
& \lesssim\|f\|_{H^{\infty}}^{q}\|g\|_{\mathcal{D}_{q-1}^{q}}^{q}+\|g\|_{H^{\infty}}^{q}\|f\|_{\mathcal{B}}^{q-p}\|f\|_{\mathcal{D}_{p-1}^{p}}^{q-p}<\infty .
\end{aligned}
$$

Thus, $g f \in \mathcal{D}_{q-1}^{q}$ and, hence, $g f \in H^{\infty} \cap \mathcal{D}_{q-1}^{q}$. Consequently, we have proved that $g \in M\left(H^{\infty} \cap \mathcal{D}_{p-1}^{p}, H^{\infty} \cap \mathcal{D}_{q-1}^{q}\right)$.

\section{Proof of Theorem 4}

Let $\tilde{p}=\min \{p, 1\}$ and $p^{\star}=\min \{p, 2\}$. We shall split the proof in two cases.

Case 1: $\mathbf{0}<\mathbf{q}<\mathbf{1}$. Take a sequence $\left\{u_{k}\right\}_{k=1}^{\infty} \in \ell^{\tilde{p}} \backslash \ell^{q}$ and let $f$ be defined by

$$
f(z)=\sum_{k=1}^{\infty} u_{k} z^{2^{k}}, \quad z \in \mathbb{D} .
$$

Then, using Proposition $\mathrm{A}$ and the fact that $\tilde{p} \leq 1$, we see that $f \in\left(\mathcal{D}_{p-1}^{p} \cap H^{\infty}\right) \backslash \mathcal{D}_{q-1}^{q}$. Case 2: $\mathbf{1} \leq \mathbf{q}<\mathbf{2}$. Let us consider a sequence $\left\{u_{k}\right\}$ such that $\left\{u_{k}\right\}_{k=1}^{\infty} \in \ell^{p^{\star}} \backslash \ell^{q}$ and let choose $n_{k}=4^{k}$. We claim that the function $\Phi \in H^{\infty}$ associated to $\left\{u_{k}\right\}$ and $\left\{n_{k}\right\}$ via Lemma 2 satisfies that $\Phi \in H^{\infty} \cap \mathcal{D}_{p-1}^{p} \backslash H^{\infty} \cap \mathcal{D}_{q-1}^{q}$.

Arguing as in the proof of [36, Lemma 1.6 (i) ] and bearing in mind Lemma 2 (ii), we deduce

$$
\|\Phi\|_{\mathcal{D}_{q-1}^{q}}^{q} \gtrsim \sum_{k=0}^{\infty}\left|\widehat{\Phi\left(n_{k}\right)}\right|^{q}=\left\|\left\{u_{k}\right\}\right\|_{\ell^{q}}^{q}=\infty
$$

that is, $\Phi \notin \mathcal{D}_{q-1}^{q}$.

By (1.1), if $p \geq 2$ we are done. On the other hand, if $0<p<2$ by [19, Theorem 1.1 (ii)], M. Riesz theorem and Lemma 2 (iv),

$$
\begin{aligned}
\|\Phi\|_{\mathcal{D}_{p-1}^{p}}^{q} & \leq \int_{0}^{1}(1-r)^{p-1} M_{2}^{p}\left(r, \Phi^{\prime}\right) d r \\
& \lesssim \sum_{k=0}^{\infty}\left(\left\|S_{2^{k}, 2^{k+1}} \Phi\right\|_{H^{2}}\right)^{p / 2} \\
& \lesssim \sum_{k=0}^{\infty}\left(\left\|S_{4^{k}+1,4^{k+1}+1} \Phi\right\|_{H^{2}}\right)^{p / 2} \lesssim\left\|\left\{u_{k}\right\}\right\|_{\ell^{p}}^{p}<\infty
\end{aligned}
$$

which finishes the proof.

Proof of Theorem 5(a). Assume that $0<q<1,0<q<p$ and that $g \in M\left(H^{\infty} \cap\right.$ $\left.\mathcal{D}_{p-1}^{p}, H^{\infty} \cap \mathcal{D}_{q-1}^{q}\right)$ and $g \not \equiv 0$. Take

$$
f(z)=\sum_{k=1}^{\infty} \frac{z^{2^{k}}}{k^{\frac{1}{q}}} .
$$

Then we use the Rademacher functions as in the proof of Case 3 of Theorem1(ii) to get a contradiction. Hence, $g \equiv 0$. 
Proof of Theorem 5(b). Assume that $1 \leq q<2 \leq p$. By [17, Theorem 1] there is a function $f \in H^{\infty}$ such that

$$
\int_{0}^{1}\left(1-r^{2}\right)^{q-1}\left|f^{\prime}\left(r e^{i \theta}\right)\right|^{q} d r=\infty \quad \text { for every } \theta \in B
$$

where $B$ is a subset of $[0,2 \pi]$ whose Lebesgue measure $|B|$ is $2 \pi$.

Suppose that $g \in M\left(H^{\infty} \cap \mathcal{D}_{p-1}^{p}, H^{\infty} \cap \mathcal{D}_{q-1}^{q}\right)$ and $g \not \equiv 0$. Notice that $g \in H^{\infty} \cap \mathcal{D}_{q-1}^{q}$. Since

$$
\int_{\mathbb{D}}\left(1-|z|^{2}\right)^{q-1}\left|g^{\prime}(z) f(z)\right|^{q} d A(z) \leq\|f\|_{H^{\infty}}^{q}\|g\|_{H^{\infty} \cap \mathcal{D}_{q-1}^{q}}^{q}<\infty
$$

it follows that

$$
\int_{\mathbb{D}}\left(1-|z|^{2}\right)^{q-1}\left|g(z) f^{\prime}(z)\right|^{q} d A(z)<\infty
$$

Since $g \in H^{\infty}$ and $g \not \equiv 0$, there is a set $A=A(g) \subset[0,2 \pi]$ with $|A|>0$ and such that $\lim _{r \rightarrow 1^{-}} g\left(r e^{i \theta}\right) \neq 0$ if $\theta \in A$. Then, for every $\theta \in A \cap B$ there is $r_{0}(\theta) \in(0,1)$ such that $K=\inf _{r_{0}<r<1}\left|g\left(r e^{i \theta}\right)\right|>0$. Then

$$
\int_{0}^{1}\left(1-r^{2}\right)^{q-1}\left|g\left(r e^{i \theta}\right)\right|^{q}\left|f^{\prime}\left(r e^{i \theta}\right)\right|^{q} d r \geq K^{q} \int_{r_{0}}^{1}\left(1-r^{2}\right)^{q-1}\left|f^{\prime}\left(r e^{i \theta}\right)\right|^{q} d r=\infty
$$

since $|A \cap B|>0$, this is in contradiction with (4.2). Thus $g$ must be identically 0 . This finishes the proof.

\section{Some BAsic Results on the SPACE $B M O A_{\log }$}

We shall start this section by proving some embedding relations between $B M O A_{\log }$, $\mathcal{B}_{\log }$ and $B M O A$. With this aim, we recall that $g \in B M O A_{\log }$ if and only if

$$
\sup _{a \in \mathbb{D}} \frac{\log ^{2} \frac{2}{1-|a|}}{1-|a|} \int_{S(a)}\left|g^{\prime}(z)\right|^{2}\left(1-|z|^{2}\right) d A(z)<\infty
$$

where $S(a)$ is the Carleson box associated to the interval

$$
I_{a}=\left\{e^{i t} \in \mathbb{T}:\left|\arg \left(a e^{-i t}\right)\right|<\frac{1-|a|}{2}\right\}, a \in \mathbb{D} \backslash\{0\}, \quad I_{0}=\mathbb{T} .
$$

Proposition 4. If $1>\beta>\frac{1}{2}$, then $B M O A_{\log } \subsetneq \mathcal{B}_{\log } \subsetneq \mathcal{B}_{\text {log }, \beta} \subsetneq B M O A$.

Proof. First, we prove that $B M O A_{\log } \subset \mathcal{B}_{\log }$. Take $f \in B M O A_{\log }$. Let $a \in \mathbb{D}$ and assume without loss of generality that $|a|>\frac{1}{2}$. Set $a^{\star}=\frac{3|a|-1}{2} e^{i \arg a}$ so that the disc $D\left(a, \frac{1-|a|}{2}\right)$ of center $a$ and radius $\frac{1-|a|}{2}$ is contained in the Carleson box $S\left(a^{\star}\right)$. This inclusion together with the subharmonicity of $\left|f^{\prime}\right|^{2}$ and the fact that $(1-|z|) \asymp(1-|a|)$ 
for $z \in D\left(a, \frac{1-|a|}{2}\right)$ gives

$$
\begin{aligned}
\left(\log \frac{2}{1-|a|}\right)^{2} & (1-|a|)^{2}\left|f^{\prime}(a)\right|^{2} \lesssim\left(\log \frac{2}{1-|a|}\right)^{2} \int_{D\left(a, \frac{1-|a|}{2}\right)}\left|f^{\prime}(z)\right|^{2} d A(z) \\
& \asymp \frac{\left(\log \frac{2}{1-|a|}\right)^{2}}{1-|a|} \int_{D\left(a, \frac{1-|a|}{2}\right)}\left(1-|z|^{2}\right)\left|f^{\prime}(z)\right|^{2} d A(z) \\
& \asymp \frac{\left(\log \frac{2}{1-\left|a^{\star}\right|}\right)^{2}}{1-\left|a^{\star}\right|} \int_{D\left(a, \frac{1-|a|}{2}\right)}\left(1-|z|^{2}\right)\left|f^{\prime}(z)\right|^{2} d A(z) \\
& \lesssim \frac{\left(\log \frac{2}{1-\left|a^{\star}\right|}\right)^{2}}{1-\left|a^{\star}\right|} \int_{S\left(a^{\star}\right)}\left(1-|z|^{2}\right)\left|f^{\prime}(z)\right|^{2} d A(z),
\end{aligned}
$$

so $f \in \mathcal{B}_{\log }$.

Now, let us see that the inclusion is strict. We borrow ideas from 31, Proposition 5.1 (D)]. Assume on the contrary to the assertion that $B M O A_{\log }=\mathcal{B}_{\log }$. By [22, Theorem 1] (see also [1]) there are $g_{1}, g_{2} \in \mathcal{B}_{\log }$ such that

$$
\left|g_{1}^{\prime}(z)\right|+\left|g_{2}^{\prime}(z)\right| \gtrsim \frac{1}{(1-|z|) \log \frac{2}{1-|z|}}, \quad z \in \mathbb{D} .
$$

Then, for any $a \in \mathbb{D}$

$$
\begin{aligned}
& \int_{S(a)} \frac{1}{(1-|z|) \log ^{2} \frac{2}{1-|z|}} d A(z) \lesssim \int_{S(a)}\left(\left|g_{1}^{\prime}(z)\right|+\left|g_{2}^{\prime}(z)\right|\right)^{2}\left(1-|z|^{2}\right) d A(z) \\
& \lesssim \int_{S(a)}\left|g_{1}^{\prime}(z)\right|^{2}\left(1-|z|^{2}\right) d A(z)+\int_{S(a)}\left|g_{2}^{\prime}(z)\right|^{2}\left(1-|z|^{2}\right) d A(z) \\
& \lesssim \frac{(1-|a|)}{\log ^{2} \frac{2}{1-|a|}}
\end{aligned}
$$

so bearing in mind that

$$
\int_{S(a)} \frac{1}{(1-|z|) \log ^{2} \frac{2}{1-|z|}} d A(z) \asymp \frac{(1-|a|)}{\log \frac{2}{1-|a|}},
$$

and letting $|a| \rightarrow 1^{-}$, we obtain a contradiction.

Assume now that $\beta \in\left(\frac{1}{2}, 1\right)$. Then it is clear that $\mathcal{B}_{\log } \subsetneq \mathcal{B}_{\text {log }, \beta}$. Furthermore, $f(z)=\sum_{k=1}^{\infty} \frac{z^{2^{k}}}{k^{\beta}} \in \mathcal{B}_{\text {log }, \beta} \backslash \mathcal{B}_{\text {log }}($ see [32, p. 20])

The inclusion $\mathcal{B}_{\log , \beta} \subsetneq B M O A$, for $\beta>\frac{1}{2}$, follows easily using the characterization of $B M O A$ in terms of Carleson measures (see [14, p.669]). Finally, we observe that $f(z)=\log \frac{1}{1-z} \in B M O A \backslash \mathcal{B}_{\log , \beta}$ for any $\beta>0$. This concludes the proof.

Next we find a simple sufficient condition for the membership a a function $f \in \mathcal{H}$ ol $(\mathbb{D})$ in the space $B M O A_{\log }$.

Proposition 5. Let $f$ be an analytic function in $\mathbb{D}$. If

$$
\int_{0}^{1}(1-r)\left(\log \frac{1}{1-r}\right)^{2}\left[M_{\infty}\left(r, f^{\prime}\right)\right]^{2} d r<\infty
$$

then $f \in B M O A_{\log }$. 
Proof. Suppose that $f$ satisfies (5.1) Let $I$ be an interval in $\mathbb{T}$ of length $h$, say $I=\left\{e^{i t}\right.$ : $\left.\theta_{0}<t<\theta_{0}+h\right\}$. Then

$$
\begin{gathered}
\frac{\left(\log \frac{2}{|I|}\right)^{2}}{|I|} \int_{S(I)}\left(1-|z|^{2}\right)\left|f^{\prime}(z)\right|^{2} d A(z) \asymp \frac{\left(\log \frac{2}{h}\right)^{2}}{h} \int_{1-h}^{1} \int_{\theta_{0}}^{\theta_{0}+h}(1-r)\left|f^{\prime}\left(r e^{i t}\right)\right|^{2} d r \\
\lesssim\left(\log \frac{2}{h}\right)^{2} \int_{1-h}^{1}(1-r)\left[M_{\infty}\left(r, f^{\prime}\right)\right]^{2} d r \leq \int_{1-h}^{1}(1-r)\left[M_{\infty}\left(r, f^{\prime}\right)\right]^{2}\left(\log \frac{2}{1-r}\right)^{2} d r \\
\leq \int_{0}^{1}(1-r)\left[M_{\infty}\left(r, f^{\prime}\right)\right]^{2}\left(\log \frac{2}{1-r}\right)^{2} d r .
\end{gathered}
$$

Now we turn to the question of finding conditions on the Taylor coefficients of a function $f \in \mathcal{H o l}(\mathbb{D})$ enough to assert that $f \in B M O A_{\log }$. We shall need two lemmas. The first one estimates an integral which may be viewed as a generalization of the classical beta function (compare with Lemma 2 of [10]) and we omit its proof.

Lemma 2. Whenever $m=1,2,3, \ldots$ and $\alpha>0$, we have

$$
\int_{0}^{1} x^{n}(1-x)^{m}\left(\log \frac{1}{1-x}\right)^{\alpha} d x \asymp \frac{(\log n)^{\alpha}}{n^{m+1}}, \quad \text { as } n \rightarrow \infty \text {. }
$$

Lemma 3. Suppose that $\alpha>0$ and let $g$ be an analytic function in $\mathbb{D}, g(z)=\sum_{n=0}^{\infty} a_{n} z^{n}$ $(z \in \mathbb{D})$. The following two conditions are equivalent:

(i) $\int_{\mathbb{D}}\left(1-|z|^{2}\right)\left|g^{\prime}(z)\right|^{2}\left(\log \frac{2}{1-|z|}\right)^{\alpha} d A(z)<\infty$.

(ii) $\sum_{n=1}^{\infty}\left|a_{n}\right|^{2}[\log n]^{\alpha}<\infty$.

Proof. We have

$$
\begin{gathered}
\int_{\mathbb{D}}\left(1-|z|^{2}\right)\left|g^{\prime}(z)\right|^{2}\left(\log \frac{2}{1-|z|}\right)^{\alpha} d A(z) \asymp \int_{0}^{1} r(1-r) M_{2}\left(r, g^{\prime}\right)^{2}\left(\log \frac{2}{1-|z|}\right)^{\alpha} d r \\
=\sum_{n=1}^{\infty} n^{2}\left|a_{n}\right|^{2} \int_{0}^{1}(1-r) r^{2 n-1}\left(\log \frac{2}{1-|z|}\right)^{\alpha} d r
\end{gathered}
$$

Now, using Lemma2 with $m=1$ we see that $\int_{0}^{1}(1-r) r^{2 n-1}\left(\log \frac{2}{1-|z|}\right)^{\alpha} d r \asymp \frac{[\log n]^{\alpha}}{n^{2}}$. Then it follows that $\int_{\mathbb{D}}\left(1-|z|^{2}\right)\left|g^{\prime}(z)\right|^{2}\left(\log \frac{2}{1-|z|}\right)^{2} d A(z) \asymp \sum_{n=1}^{\infty}\left|a_{n}\right|^{2}[\log (n+1)]^{\alpha}$.

We close this section proving Proposition 1.

Proof of Proposition 1. Suppose that $\sum_{k=0}^{\infty}\left|a_{k}\right|^{2}\left(\log n_{k}\right)^{3}<\infty$ and

$$
f(z)=\sum_{k=0}^{\infty} a_{k} z^{n_{k}}(z \in \mathbb{D}) \text { with } n_{k+1} \geq \lambda n_{k} \text { for all } k \text {, and } \lambda>1 .
$$

Using the Cauchy-Schwarz inequality and the fact that $\sum_{k=0}^{\infty} r^{2 n_{k}} \lesssim \log \frac{2}{1-r}$ (because the function $h$ given by $h(z)=\sum z^{2 n_{k}}$ is a Bloch function), we see that

$$
\begin{gathered}
{\left[r M_{\infty}\left(r, f^{\prime}\right)\right]^{2} \leq\left(\sum_{k=0}^{\infty} n_{k}\left|a_{k}\right| r^{n_{k}}\right)^{2}} \\
\leq\left(\sum_{k=0}^{\infty} n_{k}^{2}\left|a_{k}\right|^{2} r^{2 n_{k}}\right)\left(\sum_{k=0}^{\infty} r^{2 n_{k}}\right) \lesssim\left(\log \frac{2}{1-r}\right) \sum_{k=0}^{\infty} n_{k}^{2}\left|a_{k}\right|^{2} r^{2 n_{k}}
\end{gathered}
$$

Then, using Lemma 2 with $m=1$ and $\alpha=3$, we obtain

$$
\begin{gathered}
\int_{0}^{1}(1-r)\left(\log \frac{1}{1-r}\right)^{2}\left[M_{\infty}\left(r, f^{\prime}\right)\right]^{2} d r \lesssim \int_{0}^{1}(1-r)\left(\log \frac{1}{1-r}\right)^{3}\left(\sum_{k=0}^{\infty} n_{k}^{2}\left|a_{k}\right|^{2} r^{2 n_{k}}\right) d r \\
=\sum_{k=0}^{\infty} n_{k}^{2}\left|a_{k}\right|^{2} \int_{0}^{1} r^{2 n_{k}}(1-r)\left(\log \frac{1}{1-r}\right)^{3} d r \lesssim \sum_{k=0}^{\infty}\left|a_{k}\right|^{2}\left(\log n_{k}\right)^{3}<\infty .
\end{gathered}
$$

Then Proposition 5 implies that $f \in B M O A_{\log }$. 
To see that $f \in H^{\infty}$ observe that $\lambda^{k} \lesssim n_{k}$ and $\left|a_{k}\right|^{2} \lesssim\left(\log n_{k}\right)^{-3}$. Then it follows that $\left|a_{k}\right|=\mathrm{O}\left(k^{-3 / 2}\right)$, as $k \rightarrow \infty$ and the result follows.

\section{RANDOM POWER SERIES}

In this section we shall consider random power series analytic in $\mathbb{D}$ of the form

$$
\sum_{n=0}^{\infty} \epsilon_{n} a_{n} z^{n}
$$

where the $\epsilon_{n}$ 's are random signs. More precisely, if $f \in \mathcal{H}$ ol $(\mathbb{D}), f(z)=\sum_{n=0}^{\infty} a_{n} z^{n}$ $(z \in \mathbb{D})$, we set

$$
f_{t}(z)=\sum_{n=0}^{\infty} r_{n}(t) a_{n} z^{n}, \quad 0 \leq t<1, \quad z \in \mathbb{D},
$$

where the $r_{n}$ 's are the Rademacher functions. Each function $f_{t}$ is analytic in $\mathbb{D}$. Littlewood 25] (see also [9, Appendix A]) proved that if $\sum_{n=0}^{\infty}\left|a_{n}\right|^{2}<\infty$ then $f_{t} \in \cap_{0<p<\infty} H^{p}$ almost surely (a.s.), that is, for almost every $t$. On the other hand, the condition $\sum_{n=0}^{\infty}\left|a_{n}\right|^{2}=\infty$ implies that for almost every $t, f_{t}$ has a radial limit almost nowhere.

Paley and Zygmund [29] gave an example of an $f$ with

$$
\sum_{n=1}^{\infty}\left|a_{n}\right|^{2} \log n<\infty
$$

such that $f_{t} \notin H^{\infty}$ for every $t$.

Anderson, Clunie and Pommerenke [2] used a result of Salem and Zygmund [33. on the behaviour of the maxima of the partial sums of random trigonometric series to prove that (6.1) implies that $f_{t} \in \mathcal{B}$ a.s. and that this condition is best possible. Later on, Sledd 35 used also the Salem and Zygmund theorem to show that (6.1) actually implies that $f_{t} \in B M O A$ a.s.

Duren proved in [10] the following result.

Theorem A. If $0 \leq \beta \leq 1$ and $\sum_{n=1}^{\infty}\left|a_{n}\right|^{2}(\log n)^{\beta}<\infty$, then for almost every $t \in$ $[0,1]$, the function

$$
f_{t}(z)=\sum_{n=1}^{\infty} r_{n}(t) a_{n} z^{n}, \quad z \in \mathbb{D}
$$

satisfies

$$
\int_{0}^{1}(1-r)\left(\log \frac{1}{1-r}\right)^{\beta-1}\left[M_{\infty}\left(r, f_{t}^{\prime}\right)\right]^{2} d r<\infty
$$

Using this, Duren gave in [10] a new proof of Sledd's theorem. Next we prove an analogue of Duren's theorem for $\beta=3$. This will allow us to obtain the analogue of Sledd's theorem for $B M O A_{\log }$.

Theorem 11. If $\sum_{n=1}^{\infty}\left|a_{n}\right|^{2}(\log n)^{3}<\infty$ then for almost every $t \in[0,1]$, the function

$$
f_{t}(z)=\sum_{n=1}^{\infty} r_{n}(t) a_{n} z^{n}, \quad z \in \mathbb{D}
$$


satisfies

$$
\int_{0}^{1}(1-r)\left(\log \frac{1}{1-r}\right)^{2}\left[M_{\infty}\left(r, f_{t}^{\prime}\right)\right]^{2} d r<\infty
$$

Another result of [33] implies that the condition $\sum_{n=1}^{\infty}\left|a_{n}\right|^{2}[\log n]^{\beta}<\infty$ for some $\beta>1$, implies for almost every $t, f_{t}$ has a continuous extension to the closed unit disc. Using this, Proposition 5 , and Theorem 11 we obtain the first part of Theorem 6. Part (ii) of this theorem can be proved arguing as in section 3.4 of [2], and we omit the proof.

The proof of Theorem 11 follows the lines of that of Theorem A in [10]. We shall use the result of Salem and Zygmund already mentioned (Lemma 1 of [10]), Hilbert's inequality (Lemma 2 of [10]) and Lemma2 with $m=3$ and $\alpha=2$.

Proof of Theorem 11. Set

$$
B_{n}^{2}=\sum_{k=1}^{\infty} k^{2}\left|a_{k}\right|^{2}, \quad n=1,2, \ldots,
$$

and $\psi(r)=(1-r) \sum_{n=1}^{\infty} B_{n} \sqrt{\log n} r^{n}(0<r<1)$. Just as in p. 84 of [10], we have

$$
\left|f_{t}{ }^{\prime}(z)\right| \leq C \psi(r), \quad|z|=r, \quad 0<r<1, \quad \text { almost surely. }
$$

Using Lemma2, the simple fact that $\frac{\log x}{x^{3 / 2}}$ decreases as $x$ increases in $\left[e^{2 / 3}, \infty\right)$, and Hilbert's inequality, we deduce

$$
\begin{aligned}
& \int_{0}^{1}(1-r)\left(\log \frac{1}{1-r}\right)^{2}[\psi(r)]^{2} d r \\
& \asymp \int_{0}^{1}(1-r)^{3}\left(\log \frac{1}{1-r}\right)^{2}\left[\sum_{n=1}^{\infty} B_{n} \sqrt{\log n} r^{n}\right]^{2} d r \\
& =\sum_{n=1}^{\infty} \sum_{j=1}^{\infty} B_{n} \sqrt{\log n} B_{j} \sqrt{\log j} \int_{0}^{1} r^{n+j}(1-r)^{3}\left(\log \frac{1}{1-r}\right)^{2} d r \\
& \lesssim \sum_{n=1}^{\infty} \sum_{j=1}^{\infty} \frac{B_{n} \sqrt{\log n} B_{j} \sqrt{\log j}}{(n+j)^{4}}[\log (n+j)]^{2} \\
& \leq \sum_{n=1}^{\infty} \sum_{j=1}^{\infty} \frac{1}{n+j} \frac{B_{n}[\log n]^{3 / 2}}{n^{3 / 2}} \frac{B_{j}[\log j]^{3 / 2}}{j^{3 / 2}} \\
& \lesssim \sum_{n=1}^{\infty}\left|B_{n}\right|^{2} \frac{[\log n]^{3}}{n^{3}} .
\end{aligned}
$$

Now,

$$
\begin{aligned}
& \sum_{n=1}^{\infty}\left|B_{n}\right|^{2} \frac{[\log n]^{3}}{n^{3}}=\sum_{n=1}^{\infty} \sum_{k=1}^{n} k^{2}\left|a_{k}\right|^{2} \frac{[\log n]^{3}}{n^{3}} \\
& =\sum_{k=1}^{\infty} k^{2}\left|a_{k}\right|^{2} \sum_{n=k}^{\infty} \frac{[\log n]^{3}}{n^{3}} \asymp \sum_{k=1}^{\infty}\left|a_{k}\right|^{2}[\log k]^{3}<\infty .
\end{aligned}
$$

Then (6.4), $\left(^{*}\right)$ and $\left({ }^{*}\right)$ imply that (6.3) holds for almost every $t$, finishing the proof. 


\section{Multipliers on $\mathcal{D}_{p-1}^{p} \cap B M O A$}

In this section we shall prove our results concerning multipliers from $\mathcal{D}_{p-1}^{p} \cap B M O A$ to $\mathcal{D}_{q-1}^{q} \cap B M O A$. Let start with the following result.

Theorem 12. For any $p, q$ with $0<p, q<\infty$ we have

$$
M\left(\mathcal{D}_{p-1}^{p} \cap B M O A, \mathcal{D}_{q-1}^{q} \cap B M O A\right) \subset B M O A_{\log } \cap H^{\infty}=M(B M O A) .
$$

Proof. The proof uses arguments similar to those in that of Theorem1(i) and, hence, we shall omit some details.

Using that the family $\left\{\varphi_{a}: a \in \mathbb{D}\right\}$ is bounded in $\mathcal{D}_{\lambda-1}^{\lambda} \cap B M O A$ for all $\lambda>0$, we deduce that

$$
M\left(\mathcal{D}_{p-1}^{p} \cap B M O A, \mathcal{D}_{q-1}^{q} \cap B M O A\right) \subset H^{\infty}, \quad 0<p, q<\infty .
$$

Suppose now that $0<p, q<\infty$ and $g \in M\left(\mathcal{D}_{p-1}^{p} \cap B M O A, \mathcal{D}_{q-1}^{q} \cap B M O A\right)$. Let us use the test functions $f_{a}(a \in \mathbb{D})$ defined by

$$
f_{a}(z)=\log \frac{1}{1-\bar{a} z}, \quad z \in \mathbb{D} .
$$

It is easy to see that the family $\left\{f_{a}: a \in \mathbb{D}\right\}$ is also bounded $\mathcal{D}_{\lambda-1}^{\lambda} \cap B M O A$ for all $\lambda>0$. On the other hand, there exists an absolute constant $C>0$ such that for any arc $I \subset \partial \mathbb{D}$

$$
\frac{1}{C} \log \frac{2}{|I|} \leq\left|f_{a}(z)\right| \leq C \log \frac{2}{|I|}, \quad z \in S(I),
$$

where $a=\left(1-\frac{|I|}{2 \pi}\right) \xi$ with $\xi$ the center of $I$.

Then we have

$$
\begin{aligned}
& \frac{\log ^{2} \frac{2}{I T}}{|I|} \int_{S(I)}\left(1-|z|^{2}\right)\left|g^{\prime}(z)\right|^{2} d A(z) \leq \frac{C^{2}}{|I|} \int_{S(I)}\left(1-|z|^{2}\right)\left|f_{a}(z)\right|^{2}\left|g^{\prime}(z)\right|^{2} d A(z) \\
\lesssim & \frac{C^{2}}{|T|} \int_{S(I)}\left(1-|z|^{2}\right)\left|\left(f_{a} g\right)^{\prime}(z)\right|^{2} d A(z)+\frac{C^{2}}{|I|} \int_{S(I)}\left(1-|z|^{2}\right)\left|f_{a}^{\prime}(z)\right|^{2}|g(z)|^{2} d A(z) .
\end{aligned}
$$

Since $g \in M\left(\mathcal{D}_{p-1}^{p} \cap B M O A, \mathcal{D}_{q-1}^{q} \cap B M O A\right)$, the family $\left\{f_{a} g: a \in \mathbb{D}\right\}$ is bounded in $B M O A$ and hence $\sup _{I} \frac{C^{2}}{\mid T} \int_{S(I)}\left(1-|z|^{2}\right)\left|\left(f_{a} g\right)^{\prime}(z)\right|^{2} d A(z)<\infty$. Also, using that $g \in H^{\infty}$ and that the family $\left\{f_{a}: a \in \mathbb{D}\right\}$ is bounded in $B M O A$, we deduce that $\sup _{I} \frac{C^{2}}{|T|} \int_{S(I)}\left(1-|z|^{2}\right)\left|f_{a}^{\prime}(z)\right|^{2}|g(z)|^{2} d A(z)<\infty$. Consequently, we have that

$$
\sup _{I} \frac{\log ^{2} \frac{2}{\mid I}}{|I|} \int_{S(I)}\left(1-|z|^{2}\right)\left|g^{\prime}(z)\right|^{2} d A(z)<\infty,
$$

that is, $g \in B M O A_{\log }$.

Proof of Theorem [7. Suppose that $1<q<\infty$ and $0<p \leq q<\infty$. In view of Theorem[12, we only have to prove that $M(B M O A) \subset M\left(\mathcal{D}_{p-1}^{p} \cap B M O A, \mathcal{D}_{q-1}^{q} \cap B M O A\right)$.

Take $g \in M(B M O A)$ and $f \in B M O A \cap \mathcal{D}_{p-1}^{p}$. Then, clearly, $f g \in B M O A$. Using Lemma 1 and the closed graph theorem, we obtain

$$
\begin{aligned}
& \int_{\mathbb{D}}\left|(f g)^{\prime}(z)\right|^{q}\left(1-|z|^{2}\right)^{q-1} d A(z) \\
& \lesssim \int_{\mathbb{D}}\left|f^{\prime}(z) g(z)\right|^{q}\left(1-|z|^{2}\right)^{q-1} d A(z)+\int_{\mathbb{D}}\left|g^{\prime}(z) f(z)\right|^{q}\left(1-|z|^{2}\right)^{q-1} d A(z) \\
& \lesssim\|g\|_{H^{\infty}}^{q}\|f\|_{\mathcal{D}_{p-1}^{p} \cap B M O A}^{q}+\int_{\mathbb{D}}\left|f(z) g^{\prime}(z)\right|^{q}\left(1-|z|^{2}\right)^{q-1} d A(z) .
\end{aligned}
$$


Now, Proposition 4 implies that $g \in \mathcal{B}_{\log }$. Also, since $B M O A \subset H^{q}$, we have that $f \in H^{q}$. Then we see that the last integral in (7.1) is finite as in the proof of (3.3). Thus, we have proved that $g \in M\left(\mathcal{D}_{p-1}^{p} \cap B M O A, \mathcal{D}_{q-1}^{q} \cap B M O A\right)$ finishing the proof.

Proof of Theorem [8. Suppose that $0<q<p<\infty, q<2$ and $g \in M\left(\mathcal{D}_{p-1}^{p} \cap\right.$ $\left.B M O A, \mathcal{D}_{q-1}^{q} \cap B M O A\right)$ with $g \not \equiv 0$. Take $a_{n}=\frac{1}{n^{\lambda}}(n=1,2, \ldots)$ with

$$
\max \left(\frac{1}{2}, \frac{1}{p}\right)<\lambda \leq \frac{1}{q}
$$

and set $f(z)=\sum_{n=1}^{\infty} a_{n} z^{2^{n}}(z \in \mathbb{D})$. We have that $f \in \mathcal{D}_{p-1}^{p} \cap B M O A \backslash \mathcal{D}_{q-1}^{q}$. Then we use the Rademacher functions as in the proof of Case 3 of Theorem[1(ii) to get a contradiction. Hence, $g \equiv 0$.

Now we turn to prove Theorem 9. Let us notice that (i) follows from Theorem 11 and (ii) from Theorem[6(i). To prove (iii) we shall use the following lemma.

Lemma 4. Suppose that $0<q<2$ and $\alpha>0$. Let $f$ be an analytic function in $\mathbb{D}$ of the form $f(z)=\sum_{n=0}^{\infty} a_{n} z^{n}(z \in \mathbb{D})$, with $\sum_{n=0}^{\infty}\left|a_{n}\right|^{2}[\log n]^{\alpha}<\infty$. If $f \in B M O A_{\log } \cap H^{\infty}$ then

$$
f \in M\left(\mathcal{D}_{p-1}^{p} \cap B M O A, \mathcal{D}_{q-1}^{q} \cap B M O A\right), \quad \text { whenever } 0<p \leq q \text { and } \frac{q \alpha}{2-q}>1 .
$$

For $0<q \leq 1$, (iii) of Theorem 9 follows using (ii) and the lemma with $\alpha=3$, while, for $1<q<\infty$, it follows from Theorem 7

Proof of Lemma 4. Suppose that $f$ is in the conditions of the lemma and that $0<p \leq q$ and $\frac{q \alpha}{2-q}>1$.

Take $h \in \mathcal{D}_{p-1}^{p} \cap B M O A$. Since $B M O A_{\log } \cap H^{\infty}=M(B M O A)$, it follows that $f h \in B M O A$.

We have also

$$
\begin{gathered}
\int_{\mathbb{D}}(1-|z|)^{q-1}\left|(f h)^{\prime}(z)\right|^{q} d A(z) \\
\lesssim \int_{\mathbb{D}}(1-|z|)^{q-1}|f(z)|^{q}\left|h^{\prime}(z)\right|^{q} d A(z)+\int_{\mathbb{D}}(1-|z|)^{q-1}\left|f^{\prime}(z)\right|^{q}|h(z)|^{q} d A(z) \\
=I_{1}+I_{2} .
\end{gathered}
$$

The first summand $I_{1}$ is finite because $f \in H^{\infty}$ and $h \in \mathcal{D}_{p-1}^{p} \cap B M O A \subset \mathcal{D}_{q-1}^{q} \cap B M O A$. Let us estimate the second one $I_{2}$. Using Hölder's inequality with the exponents $\frac{2}{q}$ and $\frac{2}{2-q}$, we obtain

$$
\begin{gathered}
I_{2}=\int_{\mathbb{D}}(1-|z|)^{q-1}\left|f^{\prime}(z)\right|^{q}|h(z)|^{q} d A(z) \\
\left.=\int_{\mathbb{D}}\left|f^{\prime}(z)\right|^{q}(1-|z|)^{q / 2}\left(\log \frac{e}{1-|z|}\right)^{\frac{q \alpha}{2}}\left(\log \frac{e}{1-|z|}\right)^{-\frac{q \alpha}{2}}|h(z)|^{q} 1-|z|\right)^{\frac{q}{2}-1} d A(z) \\
\leq\left[\int_{\mathbb{D}}\left|f^{\prime}(z)\right|^{2}(1-|z|)\left(\log \frac{e}{1-|z|}\right)^{\alpha} d A(z)\right]^{q / 2}\left[\int_{\mathbb{D}} \frac{|h(z)|^{\frac{2 q}{2-q}}}{\left(\log \frac{e}{1-|z|}\right)^{\frac{q \alpha}{2-q}}(1-|z|)} d A(z)\right]^{(2-q) / q} .
\end{gathered}
$$

Using Lemma3, it follows that the first integral in the last product is finite. Now, notice that $f \in H^{\lambda}$ for all $\lambda<\infty$ to deduce

$$
\int_{\mathbb{D}} \frac{|h(z)|^{\frac{2 q}{2-q}}}{\left(\log \frac{e}{1-|z|}\right)^{\frac{q \alpha}{2-q}}(1-|z|)} d A(z) \leq\|f\|_{H^{\frac{2 q}{2-q}}}^{\frac{2 q}{2-q}} \int_{0}^{1} \frac{1}{\left(\log \frac{e}{1-r}\right)^{\frac{q \alpha}{2-q}}(1-r)} d r
$$


and this integral is finite because $\frac{q \alpha}{2-q}>1$. Thus $I_{2}<\infty$. Then we have that $f h \in \mathcal{D}_{q-1}^{q}$ and, hence, $f h \in \mathcal{D}_{q-1}^{q} \cap B M O A$. Consequently, we have proved that $f \in M\left(\mathcal{D}_{p-1}^{p} \cap\right.$ $\left.B M O A, \mathcal{D}_{q-1}^{q} \cap B M O A\right)$.

To finish the proof of Theorem 9 take $q \in(0,1 / 2)$ and let $\left\{a_{n}\right\}$ be defined as follows:

$$
a_{2^{k}}=(k+1)^{-1 / q}, \quad k=0,1, \ldots
$$

and $a_{n}=0$, if $n$ is not a power of 2 . Set

$$
f(z)=\sum_{n=0}^{\infty} a_{n} z^{n}=\sum_{k=0}^{\infty}(k+1)^{-1 / q} z^{2^{k}}, \quad z \in \mathbb{D} .
$$

It is clear that $\left\{a_{n}\right\}$ satisfies (1.11). Furthermore, for almost every $t, f_{t}$ is given by a lacunary power series, $f_{t}(z)=\sum_{k=0}^{\infty} r_{2^{k}}(t) a_{2^{k}} z^{2^{k}}$, which does not belong to $\mathcal{D}_{q-1}^{q}$ because $\sum_{k=0}^{\infty}\left|a_{2^{k}}\right|^{q}=\infty$.

Now turn to consider multipliers in $M\left(\mathcal{D}_{p-1}^{p} \cap B M O A, \mathcal{D}_{q-1}^{q} \cap B M O A\right)$ given by power series with Hadamard gaps. First we show that whenever $0<p \leq q \leq 1$ the power series with Hadamard gaps in $M\left(\mathcal{D}_{p-1}^{p} \cap B M O A, \mathcal{D}_{q-1}^{q} \cap B M O A\right)$ coincide with those in $\mathcal{D}_{q-1}^{q} \cap B M O A_{\log }$.

Theorem 13. Suppose that $0<p \leq q \leq 1$ and let $g$ be an analytic function in $\mathbb{D}$ given by a power series with Hadamard gaps. Then the following conditions are equivalent:
(a) $g \in M\left(\mathcal{D}_{p-1}^{p} \cap B M O A, \mathcal{D}_{q-1}^{q} \cap B M O A\right)$.
(b) $g \in \mathcal{D}_{q-1}^{q} \cap B M O A_{\log }$.

Proof. Since $\mathcal{D}_{p-1}^{p} \cap B M O A$ contains the constants functions, it is clear that $M\left(\mathcal{D}_{p-1}^{p} \cap\right.$ $\left.B M O A, \mathcal{D}_{q-1}^{q} \cap B M O A\right) \subset \mathcal{D}_{q-1}^{q}$ and the inclusion $M\left(\mathcal{D}_{p-1}^{p} \cap B M O A, \mathcal{D}_{q-1}^{q} \cap B M O A\right) \subset B M O A_{\log }$ follows from Theorem 12, Hence, the implication $(\mathrm{a}) \Rightarrow$ (b) holds.

Let us prove next the other implication. So take $g \in \mathcal{D}_{q-1}^{q} \cap B M O A_{\log } \cap \mathcal{L}$,

$$
g(z)=\sum_{k=0}^{\infty} a_{k} z^{n_{k}}(z \in \mathbb{D}) \text { with } n_{k+1} \geq \lambda n_{k} \text { for all } k \text {, for a certain } \lambda>1 .
$$

We have $\sum_{k=0}^{\infty}\left|a_{k}\right|^{q}<\infty$ which, since $q \leq 1$, implies that $\sum_{k=0}^{\infty}\left|a_{k}\right|<\infty$. Thus $g \in H^{\infty}$. Then $g \in B M O A_{\log } \cap H^{\infty}=M(B M O A)$.

Take $f \in \mathcal{D}_{p-1}^{p} \cap B M O A$. Since $g \in M(B M O A)$, we have that $g f \in B M O A$. Now,

$$
\begin{gathered}
\int_{\mathbb{D}}\left|(g f)^{\prime}(z)\right|^{q}\left(1-|z|^{2}\right)^{q-1} d A(z) \\
\lesssim \int_{\mathbb{D}}|g(z)|^{q}\left|f^{\prime}(z)\right|^{q}\left(1-|z|^{2}\right)^{q-1} d A(z)+\int_{\mathbb{D}}|f(z)|^{q}\left|g^{\prime}(z)\right|^{q}\left(1-|z|^{2}\right)^{q-1} d A(z) \\
=I_{1}+I_{2} .
\end{gathered}
$$

The first summand $I_{1}$ is finite because $g \in H^{\infty}$ and $f \in \mathcal{D}_{q-1}^{q}$.

Let us estimate the second one. Using Theorem 3.2 of $[20$ we see that the measure $\mu_{g, q}$ in $\mathbb{D}$ defined by $d \mu_{g, q}(z)=\left(1-|z|^{2}\right)^{q-1}\left|g^{\prime}(z)\right|^{q} d A(z)$ is a Carleson measure and (see, e.g., [37, Theorem 1] or [36. Theorem 2.1]) this implies that $\mu_{g, q}$ is a Carleson measure for $\mathcal{D}_{q-1}^{q}$, that is, $\mathcal{D}_{q-1}^{q} \subset L^{q}\left(d \mu_{g, q}\right)$. Hence $f \in L^{q}\left(d \mu_{g, q}\right)$ which is equivalent to saying that $I_{2}<\infty$. Hence, $g f \in \mathcal{D}_{q-1}^{q}$.

So, we have proved that $g f \in \mathcal{D}_{q-1}^{q} \cap B M O A$ for any $f \in \mathcal{D}_{p-1}^{p} \cap B M O A$, that is, $g \in M\left(\mathcal{D}_{p-1}^{p} \cap B M O A, \mathcal{D}_{q-1}^{q} \cap B M O A\right)$.

Finally, we obtain also the analogue of Theorem 9 for lacunary power series. 
Theorem 14. Let $f \in \mathcal{H o l}(\mathbb{D})$ be given by a lacunary power series, of the form

$$
f(z)=\sum_{k=0}^{\infty} a_{k} z^{n_{k}} \quad(z \in \mathbb{D}) \text { with } n_{k+1} \geq \lambda n_{k} \text { for all } k \text {, for a certain } \lambda>1 \text {, }
$$

and suppose that the sequence of coefficients $\left\{a_{k}\right\}_{k=0}^{\infty}$ satisfies

$$
\sum_{k=1}^{\infty}\left|a_{k}\right|^{2}\left(\log n_{k}\right)^{3}<\infty .
$$

Then the function $f$ satisfies the following conditions:

(i) $f \in M(B M O A)$

(ii) $f \in M\left(\mathcal{D}_{p-1}^{p} \cap B M O A, \mathcal{D}_{q-1}^{q} \cap B M O A\right)$ whenever $0<p \leq q$ and $q>\frac{1}{2}$.

Furthermore, if $0<q<\frac{1}{2}$ then there exists a sequence $\left\{a_{k}\right\}$ which satisfies (7.2) and such that $f \notin \mathcal{D}_{q-1}^{q}$. Thus for this sequence $\left\{a_{k}\right\}$ the function $f$ satisfies:

(a) $f \in M(B M O A)$.

(b) If $0<p \leq \lambda$ and $\lambda>1 / 2$ then $f \in M\left(\mathcal{D}_{p-1}^{p} \cap B M O A, \mathcal{D}_{\lambda-1}^{\lambda}\right)$ whenever $0<p \leq$ $\lambda$.

(c) $f \notin M\left(\mathcal{D}_{p-1}^{p} \cap B M O A, \mathcal{D}_{q-1}^{q} \cap B M O A\right)$ whenever $0<p \leq q$.

Proof. Part (i) follows from Proposition1, Part (ii) follows from Theorem 7 for $q>1$ and from Lemma 4 (with $\alpha=3$ ) whenever $0<q \leq 1$.

Now, if $0<q<\frac{1}{2}$ take

$$
a_{k}=k^{-1 / q}, \quad k=1,2, \ldots
$$

and

$$
f(z)=\sum_{k=1}^{\infty} a_{k} z^{2^{k}}, \quad z \in \mathbb{D} .
$$

Clearly,

$$
\sum_{k=1}^{\infty}\left|a_{k}\right|^{2} k^{3}<\infty, \quad \text { and } \quad \sum_{k=1}^{\infty}\left|a_{k}\right|^{q}=\infty .
$$

Then $f$ satisfies conditions (a), (b) and (c) of Theorem 14,

As we mentioned in Section 1. Theorem 14 also shows that Theorem 7 does not remain true for $q<1 / 2$.

\section{REFERENCES}

[1] E. Abakumov, and E. Doubtsov, Reverse estimates in growth estimates, Math. Z. 271 (2012), n. 1-2, 399-413.

[2] J. M. Anderson, J. Clunie and Ch. Pommerenke, On Bloch functions and normal functions, J. Reine Angew. Math. 270 (1974), 12-37.

[3] J. Arazy, Multipliers of Bloch functions, University of Haifa Mathem. Public. Series 54 (1982).

[4] A. Baernstein, Analytic functions of Bounded Mean Oscillation In: Aspects of Contemporary Complex Analysis, Editors: D. A. Brannan and J. G. Clunie., Academic Press, London, New York (1980), pp. $3-36$.

[5] A. Baernstein, D. Girela and J. A. Peláez, Univalent functions, Hardy spaces and spaces of Dirichlet type, Illinois J. Math. 48 (2004), no. 3, 837-859.

[6] L. Brown and A. Shields, Multipliers and cyclic vectors in the Bloch space, Michigan Math. J. 38 (1991), 141-146.

[7] S. M. Buckley, P. Koskela and D. Vukotić, Fractional integration, differentiation, and weighted Bergman spaces, Math. Proc. Cambridge Philos. Soc. 126 (1999), 369-385.

[8] J. Clunie and T. MacGregor, Radial growth of the derivate of univalent functions, Comment. Math. Helv. 59 (1984), 362-375. 
[9] P. L. Duren, Theory of $H^{p}$ Spaces, Academic Press, New York-London 1970. Reprint: Dover, Mineola, New York 2000.

[10] P. L. Duren, Random series and bounded mean oscillation, Michigan Math. J. 32 (1985), no. 1, 81-86.

[11] P. L. Duren and A. P. Schuster, Bergman Spaces, Math. Surveys and Monographs, Vol. 100, American Mathematical Society, Providence, Rhode Island, 2004.

[12] T. M. Flett, The dual of an inequality of Hardy and Littlewood and some related inequalities, J. Math. Anal. Appl. 38 (1972), 756-765.

[13] J. F. Fournier, An interpolation problem for coefficients of $H^{\infty}$ functions, Proc. Amer. Math. Soc., 42, n. 2, 402-408, (1974).

[14] P. Galanopoulos, D. Girela and R. Hernández, Univalent functions, VMOA and related spaces, J. Geom. Anal. 21 (2011), no. 3, 665-682.

[15] P. Galanopoulos, D. Girela and J. A. Peláez, Multipliers and integration operators on Dirichlet spaces, Trans. Amer. Math. Soc. 363 (2011), no. 4, 1855-1886.

[16] J. B. Garnett, Bounded Analytic Functions, Academic Press, New York, London, 1981.

[17] D. Girela, Growth of the derivative of bounded analytic functions, Complex Variables Theory Appl. 20 (1992), no. 1-4, 221-227.

[18] D. Girela, Analytic functions of bounded mean oscillation. In: Complex Function Spaces, Mekrijärvi 1999 Editor: R. Aulaskari. Univ. Joensuu Dept. Math. Rep. Ser. 4, Univ. Joensuu, Joensuu (2001) pp. $61-170$.

[19] D. Girela and J. A. Peláez, Growth properties and sequences of zeros of analytic functions in spaces of Dirichlet type, J. Australian Math. Soc. 80 (2006), 397-418.

[20] D. Girela and J. A. Peláez, Carleson measures for spaces of Dirichlet type, Integral Equations and Operator Theory 55 (2006), n. 3, 415-427.

[21] D. Girela and J. A. Peláez, Carleson measures, multipliers and integration operators for spaces of Dirichlet type, J. Funct. Analysis 241 (2006), n. 1, 334-358.

[22] J. Gröhn, J. A. Peláez and J. Rättyä, Jointly maximal products in weighted growth spaces, preprint (submitted). Available at http://arxiv.org/abs/1210.3318

[23] H. Hedenmalm, B. Korenblum and K. Zhu, Theory of Bergman Spaces, Graduate Texts in Mathematics, Vol. 199, Springer, New York, Berlin, etc. 2000.

[24] F. John and L. Nirenberg, On functions of bounded mean oscillation, Comm. Pure Appl. Math 14 (1961), 415-426.

[25] J. E. Littlewood, Mathematical notes (13): On mean values of power series (II), J. London Math. Math. Soc. 5 (1930), 179-182.

[26] J. E. Littlewood and R. E. A. C. Paley, Theorems on Fourier series and power series. II, Proc. London Math. Soc. 42 (1936), 52-89.

[27] M. Mateljević and M. Pavlović, $L^{p}$ behaviour of the integral means of analytic functions, Studia Math. 77 (1984), 219-237.

[28] J. M. Ortega and J. Fàbrega, Pointwise multipliers and corona type decomposition in BMOA, Ann. Inst. Fourier (Grenoble) 46 (1996), no. 1, 111-137.

[29] R. E. A. C. Paley and A. Zygmund, On some series of functions (I), Proc. Cambridge Philos. soc. 26 (1930), 337-357.

[30] M. Pavlović, Introduction to function spaces on the disk, Matematički Institut SANU, Belgrade, (2004)

[31] J. A. Peláez and J. Rättyä, Weighted Bergman spaces induced by rapidly increasing weights, Mem. Amer. Math. Soc. (to appear). Available at http://arxiv.org/abs/1210.3311

[32] J. A. Peláez and J. Rättyä, Generalized Hilbert operators on weighted Bergman spaces, preprint. Available at http://arxiv.org/abs/1210.3315

[33] R. Salem, A. Zygmund, Some properties of trigonometric Series whose terms have random signs, Acta Math. 91 (1954), 245-301.

[34] A. G. Siskakis and R. Zhao, A Volterra type operator on spaces of analytic functions, Function spaces (Edwardsville, IL, 1998), 299311, Contemp. Math., 232, Amer. Math. Soc., Providence, RI, 1999.

[35] W. T. Sledd, Random series which are BMO or Bloch, Michigan Math. J. 28 (1981), no. 3, 259-266.

[36] S. A. Vinogradov, Multiplication and division in the space of analytic functions with area integrable derivative, and in some related spaces (in russian), Zap. Nauchn. Sem. S.-Peterburg. Otdel. 
Mat. Inst. Steklov. (POMI) 222 (1995), Issled. po Linein. Oper. i Teor. Funktsii 23, 45-77, 308; translation in J. Math. Sci. (New York) 87, no. 5 (1997), 3806-3827.

[37] Z. Wu, Carleson measures and multipliers for Dirichlet spaces, J. Funct. Anal. 169 (1999), 148-163.

[38] R. Zhao, On logarithmic Carleson measures, Acta Sci. Math. (Szeged) 69 (2003), no. 3-4, 605-618.

[39] K. Zhu, Multipliers of BMO in the Bergman metric with applications to Toeplitz operators, J. Funct. Anal. 87 (1989), no. 1, 31-50.

[40] K. Zhu, Operator Theory in Function Spaces, Second Edition, Math. Surveys and Monographs, 138 (2007).

[41] A. Zygmund, Trigonometric Series, Vol I and II, Third Edition, Cambridge Univ. Press, Cambridge (1959).

Departamento de Análisis Matemático, Universidad de Málaga, Campus de Teatinos, 29071 Málaga, Spain

E-mail address: christos.ch@uma.es

Departamento de Análisis Matemático, Universidad de Málaga, Campus de Teatinos, 29071 MÁlaga, Spain

E-mail address: girela@uma.es

Departamento de Análisis Matemático, Universidad de Málaga, Campus de Teatinos, 29071 Málaga, Spain

E-mail address: japelaez@uma.es 